\title{
Die jungpleistozänen Äolianite in der südlichen Küstenzone von Israel Ein Beitrag zur zeitlich-räumlichen Klimaentwicklung
}

\author{
Karl Brunnacker, Avraham Ronen \& Wolfgang Tillmanns *)
}

Eolian feature, wind transport, clay loess, paleoclimatic development, paleopedology, soil profile,

Upper Pleistocene, Würm Glacial, heavy mineral association, size distribution, shoreline, mediterranean region, periglacial area, correlation. Israel, El-Arisch-Gaza-Region, North Alps

Kurzfassung: Zwischen Gaza und Beer Sheva treten äolische Ablagerungen auf mit küstennahem Sand (Kurkar), landeinwärts sandigem Tonlöß und schließlich Tonlöß. Gemäß der faziellen Anordnung und der Schwermineralassoziation war die eiszeitliche Küstenzone Lieferquelle des äolischen Materials. Es wurde in Zeiten der Meeresregression alsgeweht. In Zeiten der Transgression haben sich makromorphologisch sehr kräftige Böden (Hamra) gebildet, nämlich ein Bodenkomplex, der das letzte Interglazial und das frühe Würm umfaßt, ferner ein „mittelpaläolithischer Boden". Etwas weiter nördlich ist zudem ein "epipaläolithischer Bodenkomplex" vorhanden. Ein Vergleich mit dem Periglazialraum nördlich der Alpen zeigt prinzipiell die nämliche Entwicklung, wobei absolute Datierungen von Höhlensintern und Travertinen ein zeitliches Grundgerüst liefern.

\section{[Young Pleistocene Aeolianites in the Southern Coastal Plain of Israel A Contribution to the Temporal and Spatial Climatic Development]}

A bstract: Aeolian deposits appear between Gaza and Beer Sheva with near shore sand (Kurkar), landwards sandy clay-loess and finally clay-loess. According to the facial arrangement and the heavy mineral association the source of origin of the aeolian material was the coastal zone of the last glacial time. It has been drifted during the regression of the sea. Macro-morphologically very stable soils (Hamra) have been built during the transgression. This "Middle paleolithic soil complex" comprises the last Interglacial and the Early Würm. In addition an "Epipaleolithic soil complex" has been preserved a little further north. A comparison with the periglacial area north of the Alps shows fundamentally the same development, whereby absolute datings of "Höhlensinters" and travertines supply the main events of the time scale.

\section{Einführung}

Vor allem in den wärmeren Mittelmeer-Ländern, aber auch andernorts, folgen sandige Äolianite den Küstenlinien. Demgemäß treten in Israel mehrere küstenparallel verlaufende „Kurkar"-Rücken auf. Sie stehen im Verband mit „Hamra"-Böden. Nur lokal, südlich von Haifa, ist im Liegenden der jüngsten Kurkar-Folge ein Beach-rock bekannt. Nach S hin nimmt die karbonatische Verfestigung der Aolianite deutlich ab. Dennoch sprechen wir auch dort der Einfachheit halber von Kurkar-Rücken und -Decken. Die Hamra ist als makromorphologisch kräftig ausgebildeter roter bis brauner Boden entwikkelt. Tirsoide Böden kommen hinzu. Teilweise handelt es sich um Bodenkomplexe, aufgebaut aus mehreren, unmittelbar übereinanderfolgenden Hamra- und Tirs-Böden.

*) Anschrift der Verfasser: Prof. Dr. K. B runnacker, Dr. W. Tillmanns, Geologisches Institut der Universität zu Köln, Zülpicher Straße 49, D-5000 Köln 1. - Prof. Dr. A. R o n e n, University of Haifa, Haifa/Israel. 
Die Untersuchungen wurden gemeinsam vom Lehrstuhl für Eiszeitenforschung in Köln in $\mathrm{Zu}-$ sammenarbeit mit A. RoNEN und seinen Mitarbeitern von der University of Haifa, dankenswert unterstützt durch die Stiftung Volkswagenwerk, durchgeführt. Nachfolgend wird als Teil des Vorhabens die Löß-Fazies im S der Küstenzone dargestellt, wo RANGe (1925) Löß im Raum Beer Sheva erwähnte. Klimatisch ist dieser Bereich durch nachstehende Klimadaten gekennzeichnet:

Jahresmittel

Temperatur Niederschlag Zahl der Trockenmonate

Gaza

(Kurkar-Zone)

$20,1^{\circ} \mathrm{C} \quad 388 \mathrm{~mm}$

Beer Sheva

(Löß-Bereich)

$19,5^{\circ} \mathrm{C}$

$227 \mathrm{~mm}$

El Arish

(lößfreier Bereich)

$19,5^{\circ} \mathrm{C} \quad 97 \mathrm{~mm}$

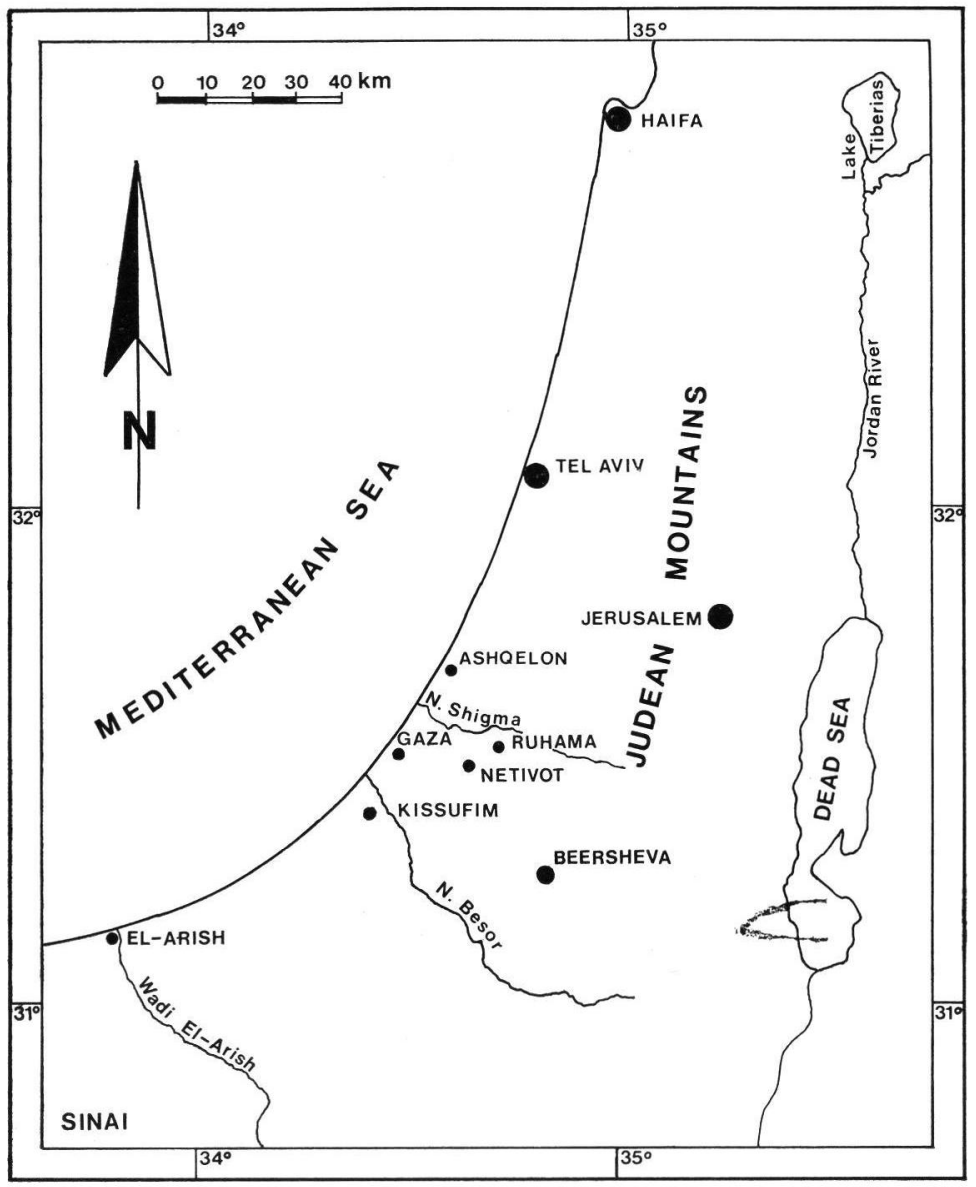

Abb. 1: Geographischer Überblick. 


\section{Profilausbildung}

Im mittleren (und z. T. im nördlichen) Küstenbereich ist folgende Gliederung der jüngeren Kurkar-Bildungen in Superposition vorhanden:

Kurkar III (im terrestrischen Bereich nur lokal)

Epipaläolithischer Bodenkomplex

Kurkar II

Mittelpaläolithischer Boden

Kurkar I

Beach-rock

Der liegende Beach-rock hat ein Th/U-Alter um 80000 Jahre (nach freundlicher Bestimmung durch Kollegen Hennig, Univ. Köln). Er vertritt damit das Neotyrrhen (STEarns \& Thurber 1965).

Im südlichen Abschnitt der Küstenzone lassen sich landeinwärts (zwischen Gaza und Beer Sheva) drei Bereiche ausscheiden, nämlich die Zone der Kurkar-Rücken, die Verzahnungszone von Kurkar mit Löß und schließlich die Löß-Zone (Abb. 1). Daneben gibt es im gesamten Gebiet nicht verfestigte subrezente bis rezente Flugsande.

\subsection{Kurkar-Zone}

Die Vorkommen liegen küstennah und gehören vorwiegend in die Gruppe der jüngeren Kurkare. Im Gebiet nördlich und nordöstlich von Gaza sind fünf mit Entfernung von der Küste zunehmend ältere Kurkar-Kerne auszumachen (Abb. 2). Ihre maximale Höhe steigt von rd. $30 \mathrm{~m} \mathrm{NN} \mathrm{(Rücken} 1$ im W) auf rd. $125 \mathrm{~m} \mathrm{NN} \mathrm{im} \mathrm{E} \mathrm{(Rücken} \mathrm{5)} \mathrm{an.}$

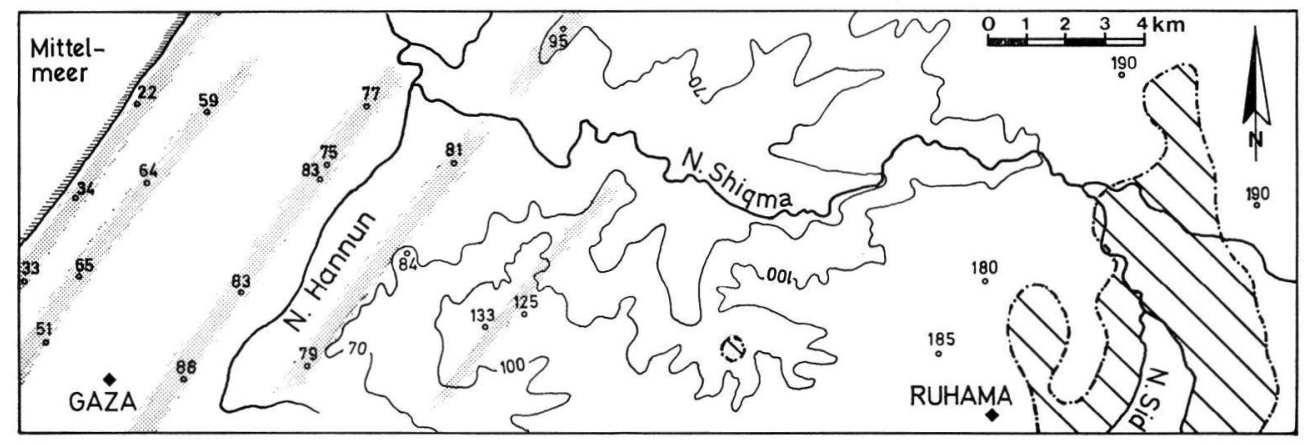

Abb. 2: Kurkar-Rücken (gerastert) in der südlichen Küstenzone von Israel (75 = Höhenangabe in $\mathrm{m} \mathrm{NN}$; Schrägschraffur $=$ Löß-Verbreitung).

Die karbonatischen, unterschiedlich stark verfestigten, z. T. groben Sande der KurkarRücken sind durch eine mit dem Alter der Rücken zunehmende Zahl von Böden (maximal 5 sandige Hamras im Rücken 5) gegliedert. Der Verfestigungsgrad der Kurkar-Sande nimmt von $\mathrm{W}$ nach $\mathrm{E}$ mit dem Alter generell zu. 


\subsubsection{Profilbeschreibung}

Typisch für die Profilausbildung in dieser Zone ist der Aufbau des küstennächsten Rückens:

$$
\text { Gabaliya (nördlich Gaza) }
$$

e) bis $700 \mathrm{~cm}$

d) $\quad 35 \mathrm{~cm}$

$100 \mathrm{~cm}$

c) $35 \mathrm{~cm}$

$50 \mathrm{~cm}$

b) $\quad 40 \mathrm{~cm}$

$100 \mathrm{~cm}$

$100-800 \mathrm{~cm}$

a) $50 \mathrm{~cm}$

$200 \mathrm{~cm}$

b) bis $150 \mathrm{~cm}$

$300-1000 \mathrm{~cm}$

a) bis $40 \mathrm{~cm}$

bis $100 \mathrm{~cm}$

$200 \mathrm{~cm}$
Kurkar, sehr schwach verfestigt

humoser, brauner Sand

Kurkar, sehr schwach verfestigt

humoser, brauner Sand

Kurkar, sehr schwach verfestigt

humoser, brauner Sand

brauner Sand

Kurkar, sehr schwach verfestigt

schwach humoser, brauner Sand

Kurkar, sehr schwach verfestigt

\section{N-Grenze Gaza-Distrikt}

rötlicher Boden

grobkörniger Kurkar, leicht verfestigt

humoser, brauner Sand

brauner Sand (z. T. verlagert, mit Gehäusen terrestrischer Mollusken) grobsandiger Kurkar, leicht verfestigt

In diesem südlichen Bereich ist das Grundprinzip der Kurkar-Ausbildung der mittleren und nördlichen Küstenzone z. T. durch die Bodenausbildung modifiziert. Die Altersstellung beider Profile ist damit allein aus der Sicht der Profilausbildung nicht genau festlegbar, wenngleich die in $\mathrm{Abb} .10$ gebrachte Einstufung naheliegend ist.

Generell ist im südlichen Kurkar-Bereich im Vergleich zum nördlichen Gebiet eine geringere Verfestigung vorhanden, was neben niedrigeren Kalkgehalten mit dem während der Eiszeiten trockeneren Klima zusammenhängt (BRUNNACKER 1979). Die fossilen Böden im Kurkar sind z. T. als Braunerden entwickelt, d.h. ein Ah-Horizont ist sehr häufig vorhanden, unter ihm liegt ein brauner Bv-Horizont. Man sollte erwarten, daß bei trokkenerem Klima eher rote Böden vorkommen. Im N und im zentralen Bereich findet man solche rote Hamra ohne Ah-Horizont, im S hingegen z. T. eine braune Hamra mit AhHorizont. Dieser Befund entspricht den Beobachtungen im epipaläolithischen Bodenkomplex nördlich Tel Aviv insofern, als unter humosen Horizonten der Bv-Horizont bräunlich gefärbt ist. Die Färbung des Bv-Horizontes steht hier mit dem überlagernden Humushorizont in Zusammenhang ( $\mathrm{pH}$-Wert).

\subsubsection{Petrographie}

Zur schwermineralogischen Charakterisierung dieser Sande wurden aus den einzelnen Kurkar-Rücken Stichproben entnommen. Die Proben (Abb. 3) zeigen eine insgesamt einheitliche Schwermineralführung, die durch eine instabile Schwermineral-Assoziation mit durchschnittlich $85 \%$ bestimmt wird. Der Anteil metamorpher Schwerminerale (Staurolith, untergeordnet Disthen und Andalusit) schwankt um 5\%. Der Anteil stabiler 
Schwerminerale (vorwiegend Zirkon) liegt um 10\%. Innerhalb der instabilen Schwerminerale dominiert die Epidotgruppe mit durchschnittlich $35 \%$, gefolgt von grüner Hornblende mit rd. 25-30\% und Pyroxen mit 15-20\%. Der Anteil des Granats liegt konstant bei $5 \%$, der Anteil der Opaken am Gesamtschwermineralspektrum zwischen 16 und $33 \%$. Nur in der Probe 10701 aus Rücken 5 beträgt der Pyroxenanteil weniger als 10\%.

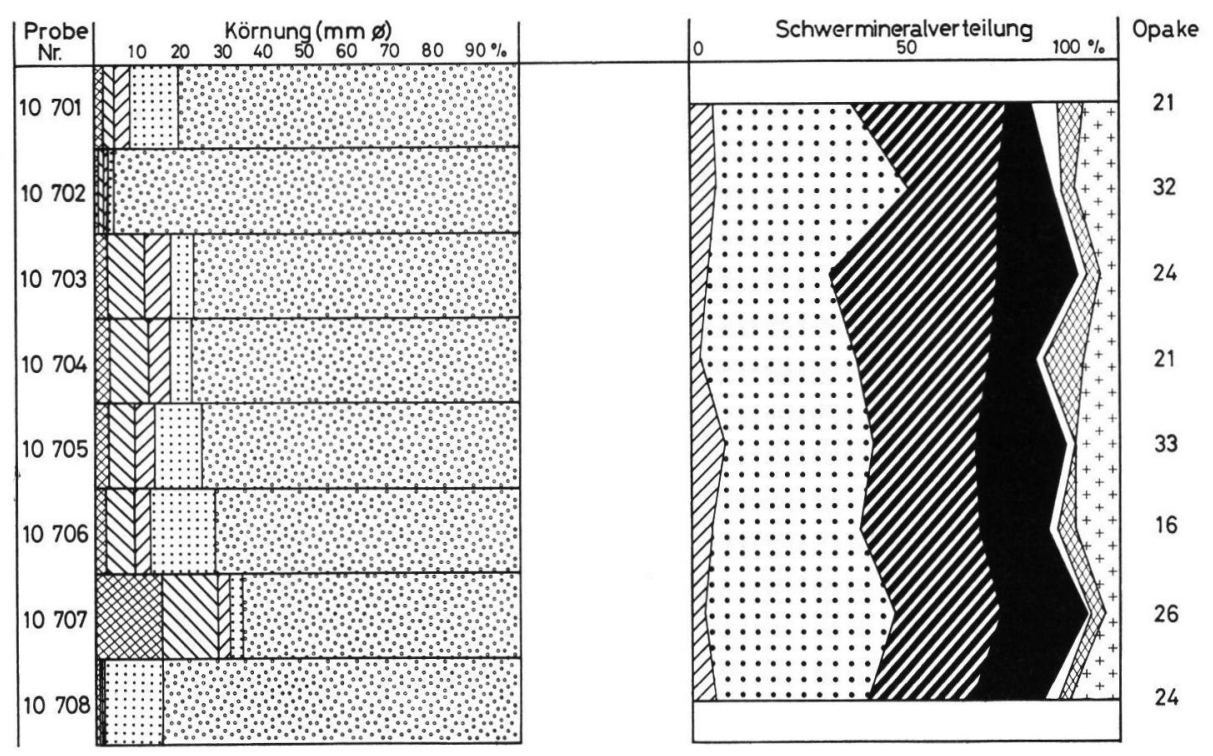

\section{Schwerminerale:}
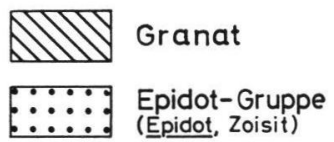

gr. Hornblende

Pyroxen (augitisch)

\section{Körnung (in $\mathrm{mm} \phi)$}

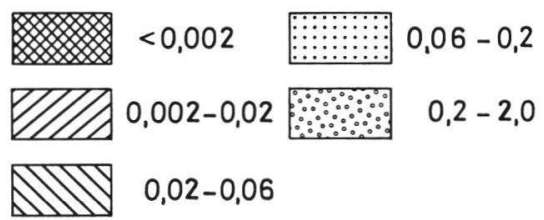

Abb. 3: Körnung und Schwermineralassoziation in der Kurkarzone.

\subsubsection{Deutung}

Qualitativ und quantitativ stimmen die Schwermineralspektren aus den Kurkar-Rükken mit den aus rezenten Küstensanden von EMERY \& NEEv (1960) beschriebenen Spektren überein. Nach den genannten Autoren handelt es sich bei den marinen Sanden um küstenparallel verfrachtete Nil-Sande. Für die Sande der Kurkar-Rücken muß, die Folgerungen aus der Lage der Rücken und deren Schrägschichtung bestätigend, ein Liefergebiet angenommen werden, das im Bereich der eiszeitlichen Küstenzone gelegen hat. 


\subsection{Verzahnungszone von Kurkar mit Löß}

Konventionellerweise werden die äolischen Decken in der Umgebung von Beer Sheva als Löß bezeichnet. Doch gibt es Verzahnungen mit Kurkar-Decken, wie das folgende Profil zeigt.

\subsubsection{Profilbeschreibung}

Der Kibbuz Kissufim liegt rd. $15 \mathrm{~km}$ südlich von Gaza und rd. $10 \mathrm{~km}$ von der Küste entfernt. Das flachwellige Gelände fällt generell sanft nach NW zum Mittelmeer und, nächst dem Aufschluß, nach NE von 100 auf rd. 50 m NN im Einzugsgebiet des Nahal Besor ab. Zu diesem heute meist trockenen Tal ist ein Wadi-System orientiert, in welchem Fundschichten erschlossen sind, die Artefakte von Typ des Jungacheuléen führen (RoNEN et al. 1972). Hier wurde das folgende Profil aufgenommen (Abb. 4):

\section{Kissufim}

\begin{tabular}{ccr} 
i) & 1 & $50 \mathrm{~cm}$ \\
\hline h) & 2 & $80 \mathrm{~cm}$ \\
& 3 & $40 \mathrm{~cm}$ \\
& 4 & $60 \mathrm{~cm}$ \\
\hline g) & 5 & $30 \mathrm{~cm}$ \\
& 6 & $50 \mathrm{~cm}$ \\
& 7 & $30 \mathrm{~cm}$ \\
& 8 & $40 \mathrm{~cm}$ \\
\hline f) & 9 & $60 \mathrm{~cm}$ \\
& 10 bis $100 \mathrm{~cm}$ \\
& 11 & $30 \mathrm{~cm}$
\end{tabular}

hellbrauner Sand (Profilversatz um $100 \mathrm{~cm}$ )

hellgraubrauner, schluffiger Feinsand

hellbraungrauer, schluffiger Feinsand, brockig, Humus in Spuren (?)

hellgraubrauner, feinsandiger Schluff

schwach humushaltiger, graubrauner, schluffiger Sand, schwach bröckelig, einzelne Kalkkonkretionen (bis $1 \mathrm{~cm} \phi$ )

humushaltiger, dunkelbraungrauer, schluffiger Sand, schwach bröckelig, zahlreiche Kalkkonkretionen (bis $1 \mathrm{~cm} \phi$ )

sehr schwach humushaltiger, graubrauner Sand, zahlreiche Kalkkonkretionen (bis $1 \mathrm{~cm} \phi$ )

hellgraubrauner Sand, zahlreiche Kalkkonkretionen (bis $3 \mathrm{~cm} \phi$ )

hellbrauner Sand, schwach hellgrau-fleckig (bis $4 \mathrm{~cm} \phi$ )

hellgraubrauner Sand, sehr schwach hellgraufleckig (bis $3 \mathrm{~cm} \phi$ )

hellgraubrauner Sand, hellgrau-fleckig (bis $5 \mathrm{~cm} \phi$ ), zahlreiche Kalkkonkretionen $(3 \mathrm{~cm} \phi)$, lagig angeordnet, unten $5 \mathrm{~cm}$ dicker rötlichbrauner Saum, an der Basis einzelne Gerölle $(3 \mathrm{~cm} \phi)$

e) $12 \quad 30 \mathrm{~cm}$

rötlichbrauner, lehmig-sandiger Ton, prismatisch-brockig, sehr viele Kalkkonkretionen (bis $4 \mathrm{~cm} \phi$ )

$1355 \mathrm{~cm}$ rötlichbrauner, lehmig-sandiger Ton, stark prismatisch-brockig, Tonbelag auf den Bodenkörpern, einzelne Kalkkonkretionen (bis $3 \mathrm{~cm} \varnothing$ )

d) $1440 \mathrm{~cm}$ rötlichbrauner, lehmiger Ton, graustichig, prismatisch-brockig, zahlreiche Kalkkonkretionen (bis $5 \mathrm{~cm} \phi$ )

$1560 \mathrm{~cm}$ rötlichbrauner, schwach lehmiger Ton, graustichig, stark prismatisch-brockig, rötlichbrauner, schwach lehmiger Ton, graustichig, stark prismatisch-bro

$1645 \mathrm{~cm}$ brauner, sandiger, lehmiger Ton, grobprismatisch (einzelne Kalkkonkretionen (bis $2 \mathrm{~cm} \phi$ )

$1780 \mathrm{~cm}$ brauner, toniger, schluffiger Sand, schwach grobprismatisch, sehr wenige Kalkkonkretionen (bis $2 \mathrm{~cm} \phi$ )

c) $18 \quad 40 \mathrm{~cm}$

dunkelbrauner, toniger, schluffiger Sand, prismatisch-brockig, zahlreiche Kalkkonkretionen (bis $3 \mathrm{~cm} \phi$ )

$1950 \mathrm{~cm}$ brauner, schwach toniger, schluffiger Sand, schwach rostfleckig, einige Kalkkonkretionen (bis $2 \mathrm{~cm} \phi$ )

b) 20 über

$22200 \mathrm{~cm}$

a) 23 über

$24 \quad 150 \mathrm{~cm}$ hellrötlichbrauner Sand, sehr wenige Kalkkonkretionen (bis $2 \mathrm{~cm} \phi$ )

hellgelbgrauer Sand, sehr wenige Kalkkonkretionen (bis $2 \mathrm{~cm} \phi$ ) 
Unter Einbezug der in Abb. 4 dargestellten Analysen ergibt sich die nachfolgende Interpretation:

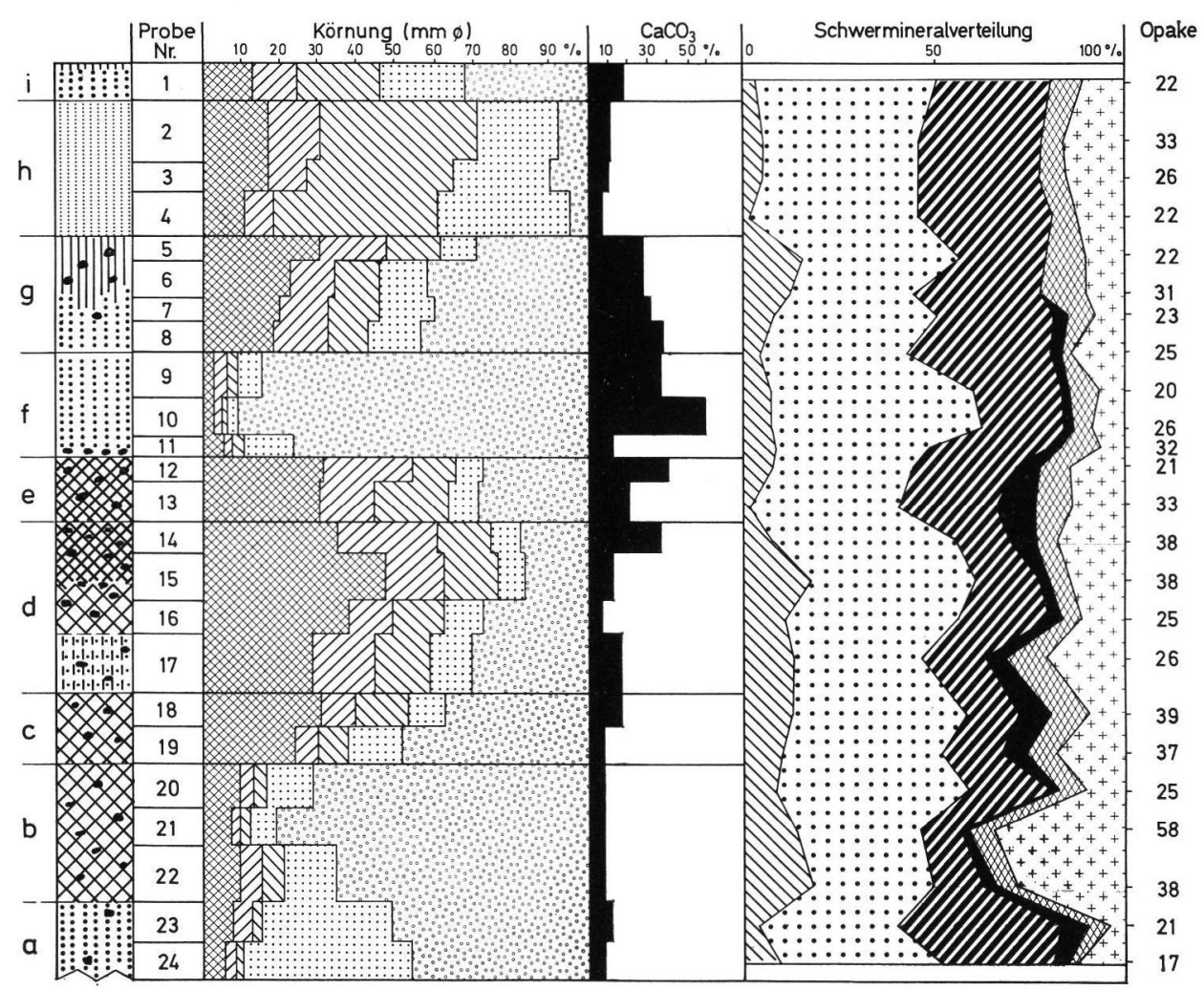

Abb. 4: Profil Kissufim (Legende Abb. 3).

Die Basis wird durch einen Kurkar-Sand (a) gebildet, der kaum verfestigt ist. Abgeschlossen wird er durch eine sandige, rote Hamra ohne Gefügemerkmale, die sich in parautochthoner Lage befindet und die Artefakte führt (b). - Die folgenden Deckschichten werden durch tonigen Sand eingeleitet, mit Abschluß durch eine braune Hamra (c). Ein körnungsmäßig als sandiger Tonlöß interpretierbares Material wird durch eine tonige, rote Hamra überprägt (d). - Die folgende geringmächtige Deckschicht entspricht in der Körnung der unterlagernden Schicht. Sie ist durch eine rotbraune Hamra völlig überformt (e). - Darüber lagert Kurkar (f), der in seiner Mächtigkeit erheblich schwankt. In den dadurch erzeugten Reliefdepressionen lagert Verschwemmungsmaterial. Es ist durch einen tirsoiden Boden überprägt (g). - Dann folgt Material mit der Körnung eines Lösses (h). - Hangenden Abschluß bildet jüngster bis rezenter Flugsand (i).

Zur zeitlichen Einstufung bringen die Artefakte in der untersten Hamra (b) generelle Hinweise. Wesentlicher ist aber beim heutigen Kenntnisstand die sedimentologisch-pedologische Interpretation des Profils. Demnach wird der liegende Kurkar (a) in die vorletzte Kaltzeit eingeordnet. Seine abschließende Hamra (b) entspricht dem letzten Interglazial. Die erhebliche pedogene Prägung der folgenden Böden (c-e) zeigt auf die frühe WürmEiszeit. Ảhnlich dem mittelpaläolithischen Boden weiter im $\mathrm{N}$ folgt über Kurkar ein tirsoider Boden ( $f-g)$. Darüber kommt lößartiges Material (h), entsprechend dem Kurkar II. Der oberste Flugsand leitet zur Gegenwart über (i). 


\subsubsection{Petrographie}

Damit stellt sich die wesentliche Frage der Liefergebiete dieser letzteiszeitlichen Sedimente, nämlich verschiedene Lieferquellen oder ein einheitliches Liefergebiet, das wegen der Verzahnung mit Kurkar-Sand letztlich nur die eiszeitliche Küstenzone gewesen sein kann. Dazu wurde das Profil schwermineralogisch untersucht (vgl. Abb. 4):

Úber das gesamte Profil hinweg dominieren instabile Schwerminerale (vorwiegend Epidot und Hornblenden, untergeordnet Granat und Pyroxen) mit durchschnittlich 75 \%. Metamorphe Schwerminerale (vorwiegend Staurolith, untergeordnet Disthen, selten Sillimanit und Andalusit) sind im gesamten Profil konstant mit rd. 10\% vertreten. Stabile Schwerminerale (vorwiegend Zirkon, untergeordnet die Rutilgruppe, selten Turmalin) sind mit durchschnittlich $15 \%$ vertreten. Nur im hangenden Teil des Kurkars und der überlagernden Hamra steigt der Anteil der Stabilen auf max. $35 \%$ an, einhergehend mit einer Reduzierung der instabilen Schwerminerale und hier im besonderen der Hornblende. In den das Profil abschließenden Löß- und Flugsand-Decken fehlen bei sonst gleichem Schwermineralspektrum die Pyroxene. Die Zahl der Opaken steigt in den pedogen überprägten Profilabschnitten bis auf maximal $58 \%$ der Gesamt-Schwerminerale an.

\subsubsection{Deutung}

Abgesehen von der erheblich wechselnden Körnungsverteilung ist insofern kein prinzipieller Unterschied zur Kurkar-Zone nördlich Tel Aviv vorhanden, als die eingeschalteten Böden sich völlig gleichen. Allerdings treten in Kissufim über den mittel- und epipaläolithischen Boden der nördlichen Zone hinaus insgesamt fünf Paläoböden auf, von denen die unteren vier zu einem Komplex zusammengeschlossen sind. Damit ist eine sehr große Ahnlichkeit mit dem Profil von Kitros in Nord-Griechenland gegeben (Brunnacker et al. 1969). Schwierig ist es aber hier wie dort, zu einer stratigraphischen Interpretation zu kommen. Wenigstens im ersten Ansatz, unabhängig von Kitros, ist es im Kissufim möglich, das oberste lößartige Glied (h) mit dem Kurkar II gleichzusetzen (Abb. 10).

Aufgrund des über das gesamte Profil einheitlichen Schwermineralspektrums ist für die Sedimente ein einheitliches Liefergebiet anzunehmen. Dafür kommt einmal die Küstenzone infrage, zum andern vorwiegend karbonatische, mesozoische und känozoische Sedimente im Hinterland der Küstenebene. Schwermineralanalysen zu beiden Liefergebieten finden sich u. a. bei EMERy \& NeEv (1960). Dies wird durch die laufenden Untersuchungen von W. Boenigk (mündl. Mitt.) bestätigt. Demnach dominieren in den Sedimenten des Hinterlandes stabile Schwermineral-Assoziationen, wohingegen die Strandsande und Küstendünen überwiegend instabile Schwerminerale mit Hornblende, Epidot und augitischen Pyroxenen zeigen. Letztere Assoziation steht qualitativ in voller Ubereinstimmung mit dem vorgestellten Schwermineralprofil von Kissufim und den küstennahen Kurkar-Rükken. Insgesamt geringfügige quantitative Abweichungen zu den von EMERY \& NeEv (1960) vorgestellten Analysen sind durch Granularvariation und Verwitterungsauslese bedingt. So finden sich in den äolischen Sedimenten gegenüber der Hornblende erhöhte Epidotgehalte. Der besonders in der intensiv pedogen überprägten Hamra (b) erhöhte Anteil an stabilen Schwermineralen ist - worauf auch Siatkine \& Pomerancblum (1958) hinweisen - durch Verwitterungsauslese bedingt. Damit wiederum korrespondiert hier die hohe Zahl an Opaken. Doch zeigt sich in den Böden oberhalb Nr. b darüber hinaus trotz der scheinbar intensiven pedogenen Prägung kein gravierender Einfluß der Verwitterungsauslese.

\subsection{Löß-Zone}

Aus diesem Bereich werden die Profile von Ruhama und Netivot im Negev behandelt. 


\subsection{1. $\mathrm{Ruham} \mathrm{a}$}

Der Kibbuz Ruhama liegt rd. 25 km östlich von Gaza (Abb. 1). Das flachwellige, badlandartige Gelände fällt von etwa $150 \mathrm{~m}$ NN nach SW zum Nahal Dorot ab. Die Lokalität Ruhama ist durch Artefaktfunde bekannt geworden.

\subsubsection{Profilaufbau}

Das Profil wurde in einem Aufschluß des badland-Bereiches aufgenommen (Abb. 5).

\section{Ruhama}

f) $120 \mathrm{~cm}$ Schluff, tonig (Löß), graugelb bis schwach gelbbraun, karbonatisch, molluskenführend

$2160 \mathrm{~cm}$ wie oben, mit Kalkpigmenten bis $1 \mathrm{~cm} \varnothing$

e) $3150 \mathrm{~cm}$ Ton, schluffig, rot-dunkelbraun, kurzprismatisch bis bröckelig, Tontapeten auf den Bodenkörpern schwach entwickelt, karbonatisch, mit Kalk-Lagen, -linsen und -konkretionen im Millimeter- bis Zentimeter-Bereich besonders entlang den Bodenkörpern

5 bis $800 \mathrm{~cm}$ Ton, siltig, dunkelbraun, prismatisch bis bröckelig, karbonatisch, im Millime6

7 ter-Bereich unterschiedlich stark sandstreifig (gelbbraun), mit Fe-Mn-Pigmenten (maximal $1 \mathrm{~cm} \phi)$, Tontapeten auf den Bodenkörpern schwach

d) $8100 \mathrm{~cm}$ Ton, siltig, rötlich-braun, braune Tontapeten auf den Bodenkörpern stärker entwickelt, mit Fe-Mn-Belegen auf den Bodenkörpern und karbonatischen Konkretionen bis $1 \mathrm{~cm} \varnothing$

c) $11.100 \mathrm{~cm}$ 12

Sand, tonig-siltig, rötlich-braun, bröckelig, schwach karbonatisch

b) $13 \quad 100 \mathrm{~cm}$

Ton, sandig-siltig, dunkelbraun, kurzprismatisch bis bröckelig, mit dunkelbraunen Tontapeten auf den Bodenkörpern, bereichsweise schwach karbonatisch, mit Fe-Mn-Pigmenten bis $0,5 \mathrm{~cm} \varnothing$

$1580 \mathrm{~cm}$ Ton, sandig-siltig, graubraun, mit rostroten bis rostbraunen Fein-Mittelsandlinsen im Zentimeter-Bereich, kalkfrei, schwach prismatisch-brockig, dunkelbraune Tontapeten auf den Bodenkörpern schwach entwickelt

Artefakt-Fundschicht über der Hamra und bis $30 \mathrm{~cm}$ in den hangenden Abschnitt hineinreichend $-----$

a) $1630 \mathrm{~cm}$ Fein-Grobsand, tonig, wie unten, ohne Kalkkonkretionen, schwach graufleckig 17 üb. $150 \mathrm{~cm}$ 18

Fein-Grobsand, tonig, rötlich bis schwach bräunlich, z. T. rot pigmentiert in gelbbrauner Matrix, schwach prismatisch-brockig, mit Fe-Mn-Konkretionen im Millimeter-Bereich, Tontapeten nur vereinzelt und schwach entwickelt, mit Kalkkonkretionen bis $1 \mathrm{~cm} \varnothing$, sonst kalkfrei

Liegendes: Kurkar (nicht aufgeschlossen)

Auch in Ruhama liegt an der Basis ein durch Hamra überprägter Kurkar (a). Oben treten Vernässungserscheinungen auf, die auf die Wirkungen der feuchten Reliefdepression deuten, in der sich nachfolgend in sumpfartigem Milieu tonreiches Material als Folge von Einschwemmungen abgelagert hat (b). Die Artefakte und Knochen liegen an der Grenze von a zu b. Darüber folgt Kurkar-Sand, der nachträglich durch eine Hamra überprägt wurde (c). Eine hangende Tonlöß-Decke wurde ebenfalls völlig durch Hamra überformt (d). Dann folgt ein mächtiges Paket von Tonlöß mit leichter synsedimentärer pedogener Gefügeprägung. Abgeschlossen wird es durch eine weitere Hamra (e). Löß mit höherem Schluffgehalt bildet das jüngste Glied (f). 


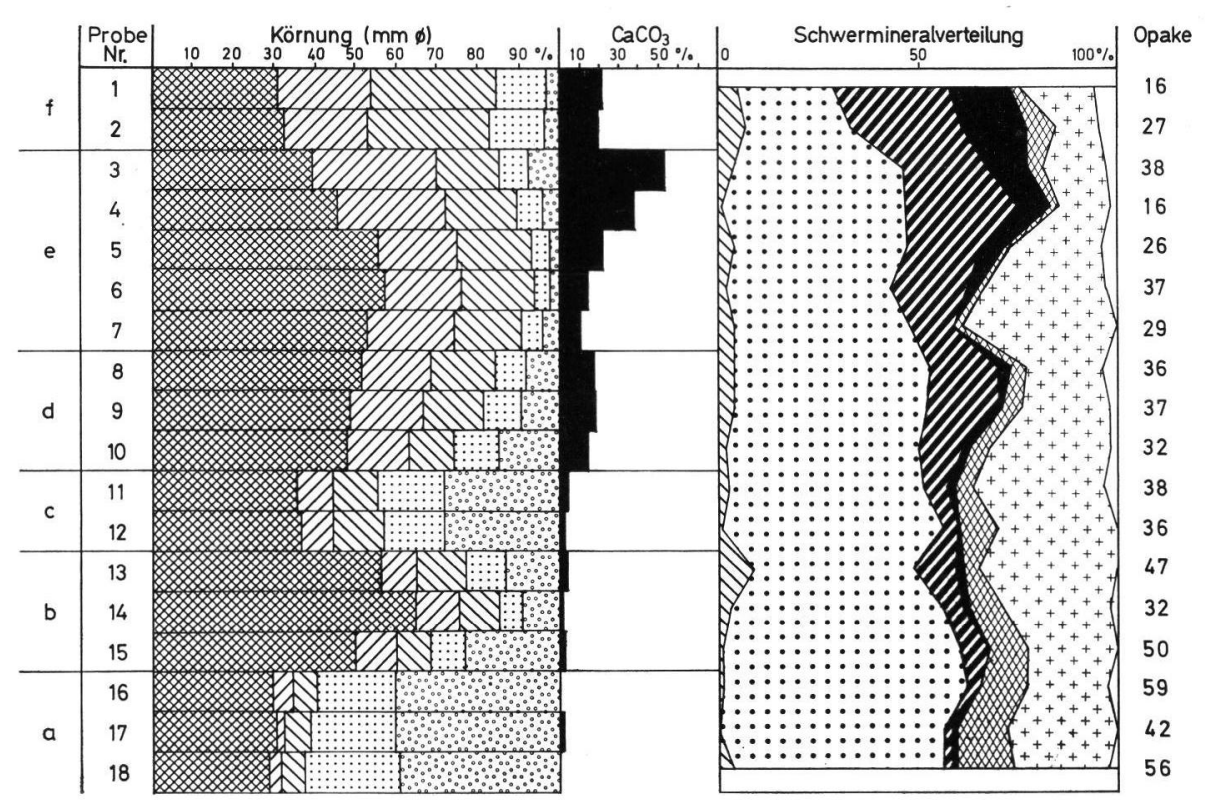

Abb. 5: Profil Ruhama (Legende Abb. 3)

Aus der Sicht der Bodenfolge ist noch eine große Ahnlichkeit mit Kissufim vorhanden, was auf eine entsprechende zeitliche Stellung weist. Wesentlich aber ist, daß in zunehmendem Maße sandige Einschaltungen zugunsten eines Tonlösses zurücktreten. Die Zunahme des Tongehaltes war schon in Kissufim bemerkbar, jedoch war dort noch ein hoher Sandanteil vorhanden, der nun in Ruhama weitgehend ausfällt. Das oberste Glied ( $g$ in Ruhama, h in Kissufim) ist jeweils durch schluffreichen Löß gekennzeichnet.

\subsubsection{Petrographie}

In allen untersuchten Proben (Abb. 5) dominieren instabile Schwerminerale (vorwiegend Epidot, daneben grüne Hornblende, untergeordnet Granat und Pyroxen) um $65 \%$. In den beiden hangenden Profilteilen nimmt der Anteil an grüner Hornblende und Pyroxen von durchschnittlich 10 auf $30 \%$ deutlich zu, gleichzeitig nimmt der Anteil der Epidotgruppe von durchschnittlich 55 auf $35 \%$ ab. Der Anteil der Metamorphen (vorwiegend Staurolith und Disthen, selten Sillimanit und Andalusit) bleibt mit Werten um 10\% über das gesamte Profil konstant. Die stabilen Schwerminerale (Zirkon, Rutilgruppe und Turmalin) zeigen bei durchschnittlichen Gehalten um 25 bis $30 \%$ in den beiden hangenden Profilabschnitten eine deutliche Abnahme, die mit einem Ansteigen insbesondere des Pyroxenanteils zusammenfällt. Die Zahl der Opaken ist besonders im liegenden Profilteil (Hamra) mit maximal 59\% relativ hoch; allgemein finden sich geringe Opakenzahlen in den pedogen wenig oder nicht beeinflußten Profilteilen.

\subsubsection{Deutung}

Das über das gesamte Profil qualitativ einheitliche Schwermineralspektrum zeigt für die Sedimente ein Liefergebiet an, das - wie beim Profil Kissufim bereits erläutert - im Bereich der Küstenzone zu suchen ist. Bezeichnend für Ruhama ist die Zunahme von grü- 
ner Hornblende und Pyroxen bei gleichzeitiger Abnahme des Anteils der Epidotgruppe und der Stabilen in den hangenden Profilabschnitten. Bei insgesamt gleicher Korngrößenverteilung kann die Granularvariation hier zur Erklärung nicht herangezogen werden.

Die niedrigen Anteile an Pyroxen und grüner Hornblende im liegenden Profilabschnitt (Hamra a-b) dürften durch eine Verwitterungsauslese bedingt sein. In Übereinstimmung damit steht der hohe Anteil an Opaken und die weitgehende Entkalkung der Sedimente dieses Bereiches. Die folgenden Profilabschnitte sind durch zunehmende Karbonatgehalte und abnehmende Opakenzahlen ausgezeichnet. $\mathrm{Da}$ diese Abschnitte $\mathrm{z}$. T. nicht oder nur schwach pedogen überprägt sind, kann eine Verwitterungsauslese keine Erklärung für die - im Vergleich zu den Liefergebieten - relativ niedrigen Anteile an grüner Hornblende und Pyroxen in den mittleren Profilabschnitten bieten. Dieser Befund ist dahingehend zu interpretieren, daß die das Profil aufbauenden Sedimente z. T. nicht direkt aus der Küstenzone geliefert wurden, sondern durch Umlagerung aus der näheren Umgebung zu beziehen sind. Ihr Schwermineralspektrum zeigt aber, daß auch diese umgelagerten Sedimente primär aus der Küstenzone geliefert wurden. Die ansteigenden Anteile an grüner Hornblende und Pyroxen in den hangenden Profilabschnitten deuten für diese eine zunehmende Beteiligung von direkt aus der Küstenzone geliefertem Material an.

\subsubsection{Netivot}

Das Profil Netivot wurde von Bruins (1976) eingehend beschrieben, worauf verwiesen sei. Es ist als Anschnitt an der Straße Gaza-Beer Sheva rd. 2 km westlich der Abzweigung nach Ashqelon bei der Ortschaft Netivot aufgeschlossen (Abb.1). Das Profil liegt in einem flachwelligen Gelände um $150 \mathrm{~m}$ NN, das nach SW zum Nahal Bohu, nach NW zum Nahal Hannun abfällt. Von der Küste ist es rd. $18 \mathrm{~km}$ entfernt.

\subsubsection{Profilaufbau}

Der rd. $400 \mathrm{~m}$ lange Aufschluß zeigt eine rd. $12 \mathrm{~m}$ mächtige Abfolge von rotbraunem, schluffigen Ton (Löß), die durch sechs ausgeprägte, bis $120 \mathrm{~cm}$ mächtige Anreicherungshorizonte von Karbonaten (vgl. BRuins 1976) gegliedert wird (Abb. 10). Der rotbraune, schluffige Ton weist ein prismatisch-brockiges Gefüge auf und zeigt auf den Bodenkörpern schwache Tontapeten.

Auf der Grundlage der Karbonathorizonte unterteilt BRuins (1976) den Tonlöß, wobei die liegende SCH 6-Schicht dem letzten Interglazial entspricht. Die folgende SCH 5- bis 2-Horizonte vertreten die Würm-Eiszeit, und SCH 1 gehört in das Spät- bis Postglazial. Doch besteht zu dieser Interpretation insofern ein prinzipieller Unterschied, als BRUINS die Karbonathorizonte den trockeneren Zeiten und wir, entsprechend BRUNNACKER (1974), als Indikatoren für etwas feuchtere Sedimentationspausen ansehen.

\subsubsection{Petrographie}

Die aus der Sicht der Korngrößenverteilung homogen ausgebildeten Sedimente zeigen auch schwermineralogisch eine über das gesamte Profil weitgehend gleiche Verteilung (Abb.6). In den Spektren dominieren instabile Schwerminerale (Epidotgruppe, grüne Hornblende, Pyroxen, untergeordnet Granat) mit durchschnittlich $75 \%$. Metamorphe Schwerminerale (Staurolith, Disthen) sind mit weniger als $5 \%$ vertreten. Bei den Stabilen, die mit durchschnittlich $25 \%$ vertreten sind, dominiert Zirkon. Bei den Instabilen nimmt der Pyroxenanteil von im Mittel 10\% im Liegenden auf 25\% im Hangenden zu, die Anteile der Epidotgruppe (um 30\%) und der grünen Hornblende (um $25 \%$ ) bleiben weitgehend konstant. Die Zunahme der Pyroxene ist mit einer Abnahme der stabilen Schwerminerale gekoppelt. Die Anteile der Opaken liegen insgesamt um 25\%, nur in den Karbonatanreicherungshorizonten SCH 5 und SCH 3 erreichen sie mit 38\% hohe Werte. 


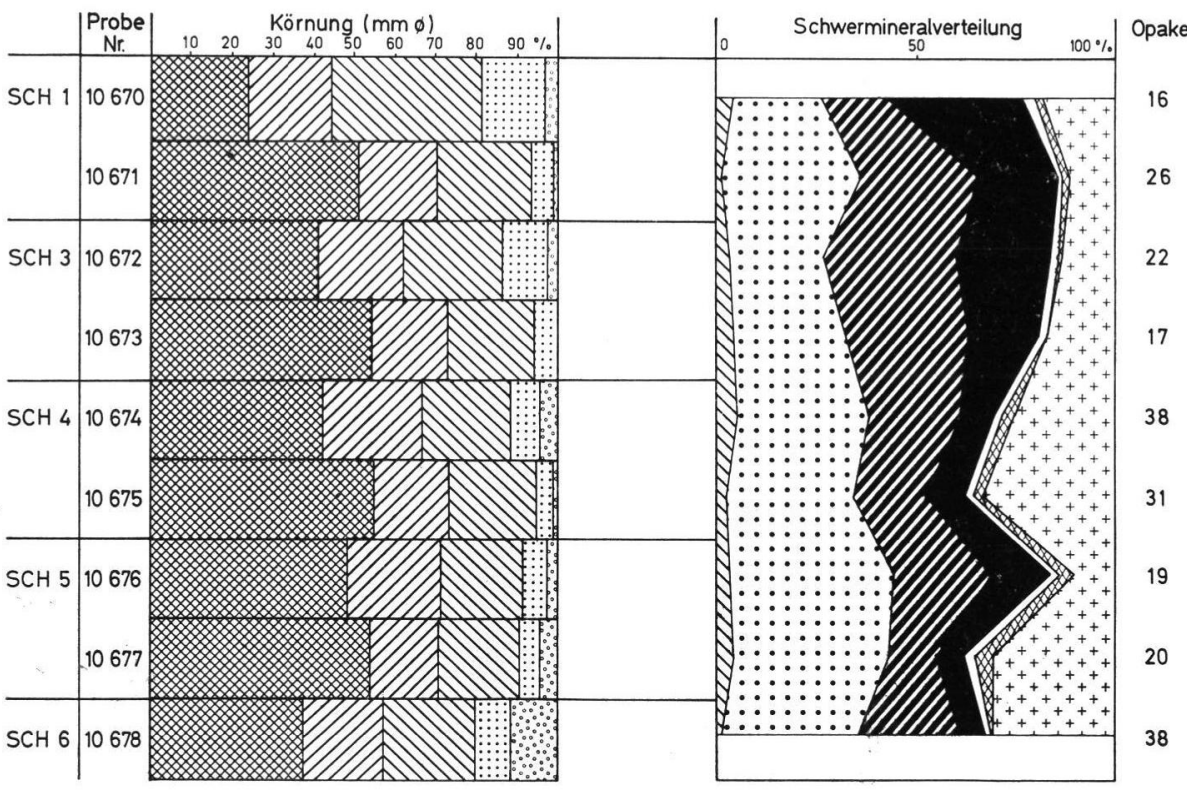

Abb. 6: Profil Netivot (vgl. Bruins 1976; Legende Abb. 3).

\subsubsection{Deutung}

Im Vergleich zu den Profilen Kissufim und Ruhama zeigt das Profil Netivot hohe Anteile an Pyroxen und grüner Hornblende. Die qualitative und quantitative schwermineralogische Zusammensetzung fällt damit weitgehend mit der solcher Profile zusammen, die aus dem Küstenbereich beschrieben werden. Demnach sollten die Sedimente entweder direkt aus dem Küstenbereich oder aber von durch Verwitterungsvorgänge nicht beeinflußten Äolianiten der Umgebung abzuleiten sein. Derartige, früher schon aus dem Küstenbereich gelieferte Äolianite haben sicher - wie auch rezent - im Negev zur Verfügung gestanden. Gegen eine solche Aufarbeitung älterer Äolianite spricht allerdings der über das gesamte Profil hinweg homogene hohe Tongehalt.

Aufgrund der Abnahme der Stabilen bei gleichzeitiger Zunahme der Pyroxene zu den hangenden Profilabschnitten hin darf angenommen werden, daß die liegenden Profilteile bis zum Karbonatanreicherungshorizont $\mathrm{SCH} 3$ relativ stärker pedogen überprägt sind.

\subsection{Ergebnis}

Lithologisch werden die beschriebenen Profile im wesentlichen aus äolischem Sand und Löß unterschiedlicher Fazies aufgebaut, wobei von der Küste landeinwärts sich ein Fazieswechsel von Sand über sandigen Tonlöß zu Tonlöß und schluffreichem Löß vollzieht. Die Sandfazies reicht in den Einzeldecken unterschiedlich weit in das Binnenland hinein. Schwermineralogisch sind keine signifikanten Unterschiede, etwa infolge Granularvariation oder verschiedener Liefergebiete, erkennbar.

Die Paläoböden, welche das Hauptkriterium für die Gliederung und Konnektierung der Profile sind, sind im allgemeinen makromorphologisch kräftig ausgebildet. Jedoch zeigen sich nur im liegenden Hamra-Boden, der in das letzte Interglazial eingestuft wird, 
deutliche Auswirkungen der Schwermineralverwitterung durch Zunahme der Opaken und Reduktion der Instabilen.

Dieser liegende Boden und die darüber folgenden nächsten drei Böden lassen sich zu einem Pedokomplex zusammenfassen, d.h. die das Solum dieser Böden bildenden äolischen oder auch fluvialen Decken sind so geringmächtig, daß sie durch die jeweils nachfolgende Pedogenese völlig überprägt wurden. Im Einzelfall ist damit aber auch denkbar, daß die eine oder andere Decke ausfällt, so daß sich dann die Wirkungen einer älteren und einer jüngeren pedogenetischen Phase addieren. Aber auch in solchen Fällen wirkt sich dies auf das Bild der Böden kaum aus.

Die jüngste pedogenetische Phase dieses Komplexes wird dem Beach-rock südlich Haifa zeitlich gleichgesetzt (Abb. 10). Mit rd. 80000 Jahren repräsentiert derselbe das Neotyrrhen. In seinem Hangenden liegen die eigentlichen Kurkar-Rücken, nämlich Kurkar I mit abschließendem mittelpaläolithischen Boden. Dieser Boden ist auch in dem hier behandelten Bereich, jedoch ohne Artefaktführung, vertreten. Im N folgt der Kurkar II. Abgeschlossen wird er z. T. durch den epipaläolithischen Boden bzw. -komplex. Die Äquivalente dieses Kurkars sind im besprochenen Gebiet ebenfalls vorhanden, z. T. mit schluffreichem Löß. Der abschließende Boden ist erodiert. Der geringmächtige Kurkar III ist auch im Norden nur lokal unmittelbar an der Küste vertreten.

\section{Materialherkunft}

Wie herausgestellt, ist von der Küste landeinwärts eine Änderung in der Körnung der äolischen Sedimente vorhanden. Dies legt generell eine Herkunft aus dem eiszeitlich-marinen Bereich nahe, in deren Folge sich eine mit der Entfernung zunehmende Kornverfeinerung einstellt. Doch steht einer solchen Interpretation die Auffassung von DaN \& YaALON (1971) und YaAlon (1969) entgegen. Demnach wurden die feinkörnigen Komponenten aus Wüstengebieten ausgeweht, insbesondere dem Sinai und Nordafrika. Zumindest also müßte demgemäß ein Gemisch aus einer marinen bis strandnahen Quelle und aus ariden Binnengebieten vorliegen. Dazu wurden im weiteren Umkreis der behandelten Profile Schwermineraluntersuchungen vorzugsweise an jungen fluviatilen Sedimenten durchgeführt (Abb. 7-9).

\subsection{Einzugsbereich ỏes Wadi el Arish}

Aus dem Einzugsbereich des Wadi el Arish (Abb. 1) wurden insgesamt 11 Sandproben schwermineralogisch untersucht (Abb. 7). Die Probennahme erfolgte

1. im Mündungsbereich des Wadi (sandige Schotter; Proben 10689 und 10690),

2. bei Bir el Lahfan (sandige Schotter; Probe 10691),

3. aus dem Nahal Lahfan zwischen Shezaf und Har Safun (sandige Schotter, Hochflutlehm und Dünensand; Proben 10692-10696) und

4. aus dem Nahal Nizzana (sandige Schotter und Dünensand; Proben 10697-10699).

In den Spektren dominieren instabile Schwerminerale (Epidotgruppe, grüne Hornblende und Pyroxen mit etwa gleichen Anteilen, untergeordnet Granat) mit im Mittel $80 \%$. Metamorphe sind mit weniger als 5\% vertreten. Der Anteil stabiler Schwerminerale ist mit durchschnittlich $15 \%$ in nahezu allen Proben gleich. Die Zahl der Opaken schwankt um $20 \%$. 


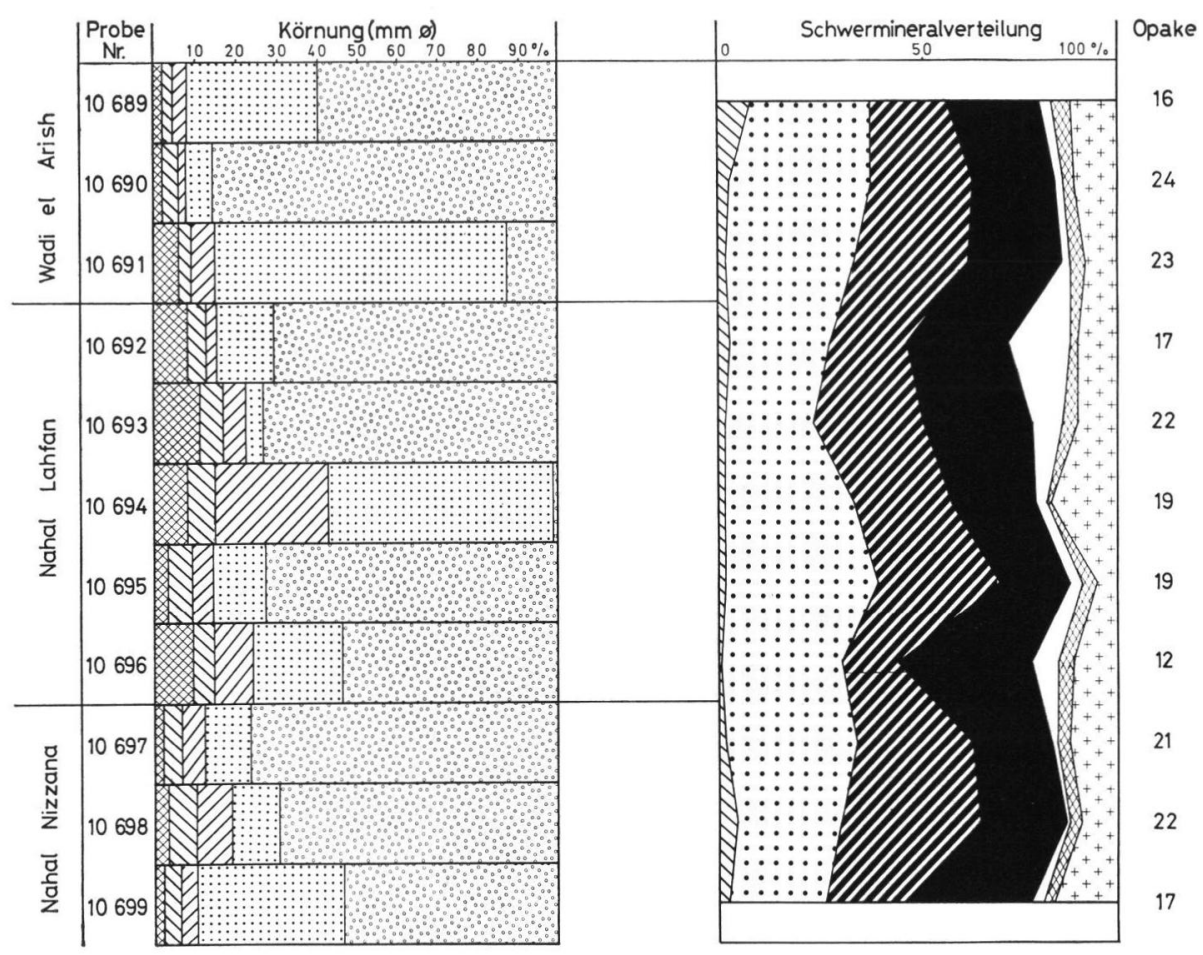

Abb. 7: Körnung und Schwermineralassoziationen im Einzugsbereich des Wadi El Arish (Legende Abb. 3).

Im Einzugsbereich des Wadi el Arish stehen kretazische und paläogene Sedimente an, die von mächtigen quartären Alluvionen und rezenten Dünen überlagert werden. Entsprechend Literaturdaten (VRoman 1944; Nachmars 1969) führen die kretazischen und paläogenen Sedimente vorzugsweise stabile Schwerminerale. Damit fallen sie als Liefergebiete für die vorgestellten Schwermineralspektren aus. Die vorgefundenen instabilen Schwermineralspektren müssen damit aus aufgearbeiteten quartären Alluvionen und rezenten Dünen abgeleitet werden, die entsprechend ihrer Schwermineralführung letztlich wiederum aus der Küstenzone abzuleiten sind. Hinweise auf eine Granularvariation, wie sie bei Fluß- und Dünensanden zu erwarten wäre, sind nicht vorhanden. Dies mag ein Kriterium auf eine sich fortwährend wiederholende Mobilisierung des Sandmaterials fluvialer und äolischer Umlagerungsprozesse sein (vgl. YAALON \& DAN 1974). Qualitativ und quantitativ zeigen die Spektren eine gute Übereinstimmung mit den hangenden Abschnitten des Profils Netivot, obwohl die dortigen Sedimente erheblich feinkörniger (Ton- und Schluffbereich) sind.

\subsection{Einzugsbereich des Nahal Besor}

Aus dem Einzugsbereich des Nahal Besor (Abb. 1) wurden insgesamt neun Sandproben schwermineralogisch untersucht (Abb. 8). Die Probennahme erfolgte

1. im Mündungsbereich bei Gaza (Ton und Sandprobe aus dem Flußbett; Proben 10679 und 10680),

2. bei Gevulot (sandige Schotter, Dünensand; (Proben 10681-10684), 
3. bei Urim (sandige Schotter; Proben 10685 und 10686)

und

4. beim Tel Gamma (sandige Schotter; Probe 10687).

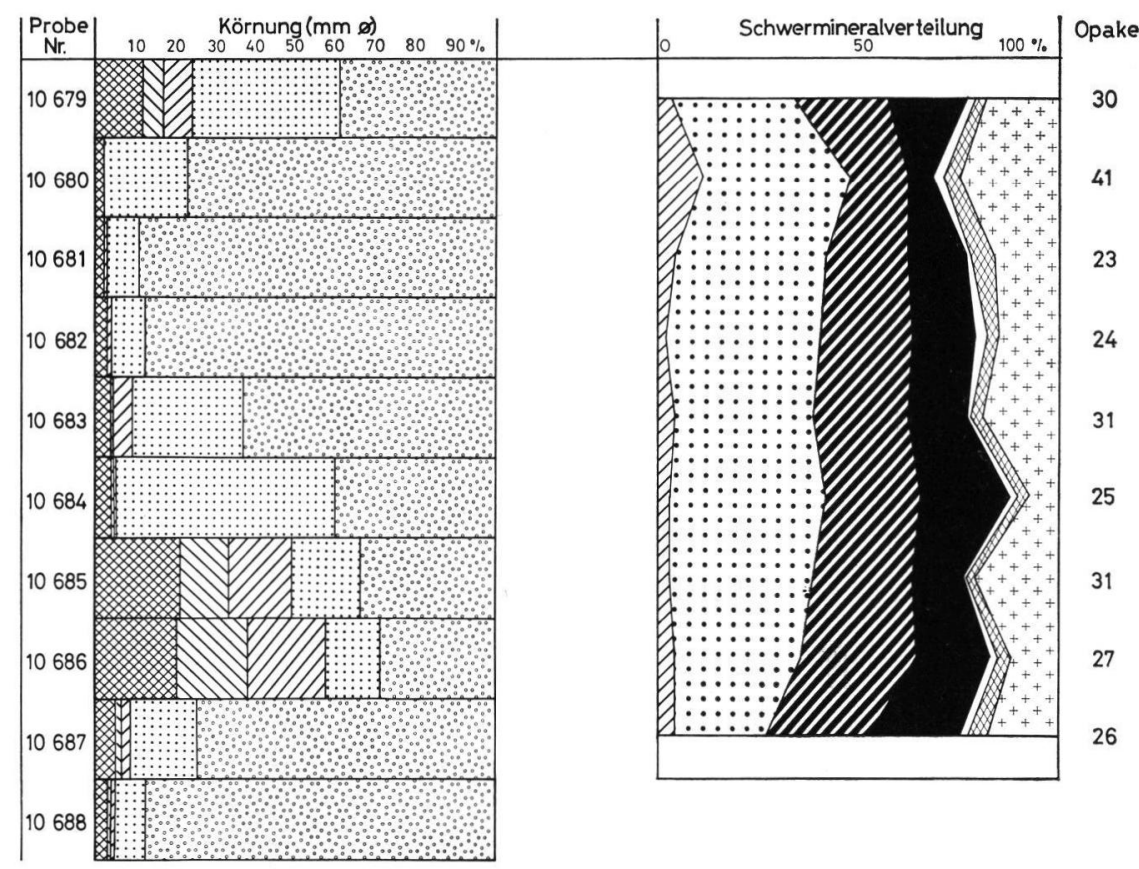

Abb. 8: Körnung und Schwermineralassoziationen im Einzugsbereich des Nahal Bezor (Legende Abb. 3).

Qualitativ und weitgehend auch quantitativ zeigen die Schwermineralspektren die gleiche Zusammensetzung wie die Proben aus dem Einzugsbereich des Wadi el Arish (Abb. 7). Bei den dominierenden instabilen Schwermineralen ist der Anteil der Pyroxene etwas geringer, der Anteil stabiler Schwerminerale ist geringfügig höher. Die Zahl der Opaken liegt mit durchschnittlich $30 \%$ etwas höher als im Bereich des Wadi el Arish.

Im Einzugsbereich des Nahal Besor ist die Schwermineralführung der fluvialen und äolischen Sedimente ebenfalls aus aufgearbeiteten quartären Alluvionen und Dünensanden abzuleiten. Eine Aufarbeitung liegender paläogener und kretazischer Sedimente spielt zumindest quantitativ keine Rolle.

\subsection{Einzugsbereich des Nahal Shiqma und des Nahal Sid}

Am Prallhang des Nahal Shiqma wurden rd. $1 \mathrm{~km}$ südlich Tel Nagila (Abb. 1) drei Proben aus einem Schotter führenden, z. T. karbonatisch verfestigten Sand und diesen überlagernden, geröll-führenden Schwemmlöß entnommen (Proben 10712-10714 — Abb. 9). Der Gerölle führende Sand überlagert diskordant oligozäne kreidige Kalke.

Am Prallhang des Nahal Sid sind rd. 4 km östlich Ruhama (Abb. 2) 3 m Oligozän (Bat guvrin-Formation), überlagert von maximal $2 \mathrm{~m}$ Schotter führenden, karbonatisch verfestigten Sandsteinen und sandigen Schottern der plio-pleistozänen Ahuzam-Formation aufgeschlossen (Proben 10709-10711). 


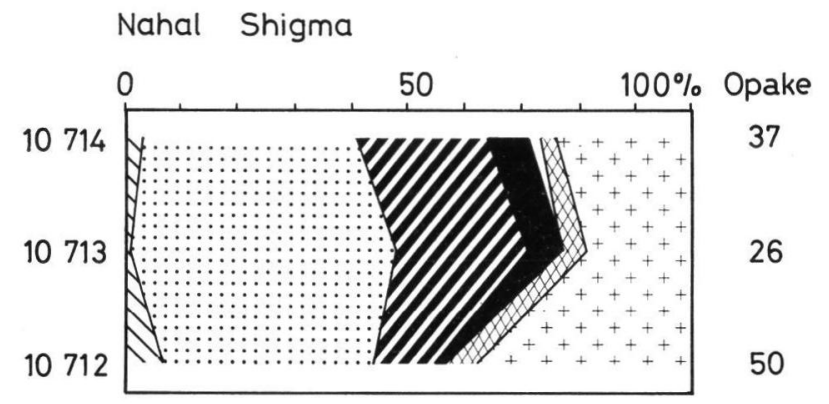

Nahal Sid

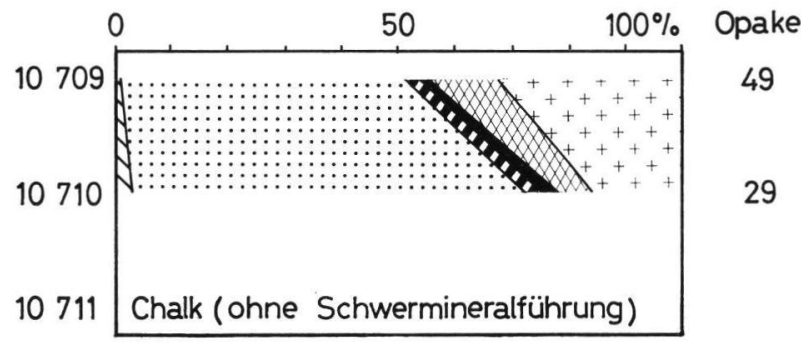

Abb. 9: Schwermineralassoziationen im Einzugsbereich des Nahal Shigma und des Nahal Sid (Legende Abb. 3).

Aus den Oligozän-Karbonaten konnten weder im Bereich des Nahal Sid noch des Nahal Shiqma Schwerminerale separiert werden. Die Schotter führenden Sande der pliopleistozänen Ahuzam-Formation (Proben 10709, 10710, 10712 - Abb. 9) zeigen ein Schwermineralspektrum, in dem instabile Schwerminerale mit Vorherrschaft der Epidotgruppe, daneben grüne Hornblende, Granat und Pyroxen mit Anteilen von 60-70\% dominieren. Der Anteil metamorpher Schwerminerale (Staurolith und Disthen) liegt um 10\%. Bei den stabilen Schwermineralen, deren Anteil um 30\% schwankt, dominiert Zirkon. Die Opakenzahl ist mit $30-50 \%$ relativ hoch. Der Gerölle führende Schwemmlöß im Hangenden der Ahuzam-Formation (Profil Nahal Shiqma, Proben 10713-10714) zeigt bei Dominanz der instabilen Schwerminerale höhere Gehalte an grüner Hornblende und Pyroxen. Der Anteil stabiler Schwerminerale geht auf durchschnittlich 20\% zurück. Die Opakenzahl ist geringer als im Liegenden.

Die oligozänen, marinen, karbonatischen Sedimente vermögen somit kaum oder keine Schwerminerale zu liefern. Die plio-pleistozänen, vorzugsweise klastisch ausgebildeten Sedimente der Ahuzam-Formation liefern vorzugsweise Schwerminerale der Epidotgruppe sowie stabile Schwerminerale. Über ihre Schwermineralführung dokumentieren diese Sedimente letztlich den Verzahnungsbereich einer sowohl marin wie auch terrestrisch beeinflußten Schüttung (NachmaIs 1969). Der hangende, Geröll führende Schwemmlöß zeigt ein von instabilen Schwermineralen bestimmtes Spektrum, wie es letztlich aus den Sedimenten der Küstenzone abzuleiten ist. Die im Vergleich zu den Küstensedimenten relativ geringen Anteile an Pyroxenen dürften durch eine Verwitterungsauslese bedingt sein. Darauf deuten auch die relativ hohen Anteile an opaken Schwermineralen. 


\subsection{Ergebnis}

Die untersuchten, pedogen nicht oder nur wenig überprägten quartären Sedimente im Bereich der Negev zeigen ein einheitliches, von Instabilen bestimmtes Schwermineralspektrum (Abb. 7-9). Die relativen Anteile der Epidotgruppe, von grüner Hornblende, Pyroxen und Granat sind in nahezu allen Proben gleich. Dies gilt sowohl für fluviatile als auch für äolische Sedimente.

Granularvariation und/oder Verwitterungsauslese machen sich in diesen Sedimenten praktisch nicht bemerkbar. Dies mag ein Hinweis auf wiederholte Umlagerungsprozesse und dadurch bedingte Homogenisierung der Sedimentkomponenten sein. Aufgrund der Schwermineralführung muß für die Sedimente ein einheitliches Liefergebiet im Bereich der Küstenzone gesucht werden. Eine Materialzufuhr aus dem von känozoischen und mesozoischen Sedimenten aufgebauten Negev-Hinterland kann wegen des geringen Anteils an stabilen Schwermineralen weitgehend ausgeschlossen werden. Nicht auszuschließen ist hingegen ein mehrphasiger Kreislauf mit Auswehung der Sedimente aus der Küstenzone, fluviatilem Rücktransport und erneuter Auswehung, worauf auch YAALON \& DAN (1974) schon hingewiesen haben.

Entsprechend den vorgestellten schwermineralogischen Befunden und ihrer Ausdeutung muß auch für die lithologisch differenzierter aufgebauten und durch Bodenbildung gegliederten Profile Kissufim, Ruhama und Netivot letztlich ein Liefergebiet im Bereich der Küstenzone gesucht werden.

Dies kommt besonders deutlich im Profil Netivot zum Ausdruck, wo zumindest die hangenden Abschnitte ein mit den Sedimenten der Küstenzone qualitativ und quantitativ übereinstimmendes Spektrum zeigen. Lediglich in den tieferen Profilabschnitten deutet sich über die geringeren Anteile an grüner Hornblende und besonders der Pyroxene bei gleichzeitiger Zunahme der stabilen Schwerminerale eine - wenn auch nur schwache - Verwitterungsauslese an. Die über das gesamte Profil einheitliche Korngrößenverteilung (schluffiger Ton) legt nahe, daß mit den Sedimenten des Profils Netivot bei entsprechend größerer Entfernung vom Auswehungsgebiet die feinkörnigere Fazies der Küstenäolianite gefaßt ist.

Die Profile Ruhama und Kissufim zeigen in bezug auf ihren lithologischen und pedogenen Aufbau eine stärkere Differenzierung, die durch Korngrößenverteilung, Kalkgehalt, Schwermineralführung und Bodenhorizonte belegt ist. Gleichwohl ist durch die qualitative Schwermineralführung nachgewiesen, daß auch diese Sedimente primär aus dem Bereich der Küstenzone zu beziehen sind. Neben Verwitterungsauslese und Granularvariation sind quantitative Anderungen im Schwermineralspektrum dadurch bedingt, daß die Sedimente z. T. nicht direkt aus der Küstenzone geliefert wurden. Umlagerungsprozesse mit Mobilisierung von offensichtlich bereits pedogen überprägtem Material aus der näheren Umgebung spielen in einzelnen Profilteilen eine bedeutende Rolle. Damit muß ein gewisser Kreislauf unterstellt werden: randmariner Auswehungsbereich - äolische Umlagerung mit Kornsaigerung - fluvialer Rücktransport - erneute Auswehung. Ob die randmarine Lieferquelle letztlich ihren Nachschub allein aus dem Nil-Delta infolge Strandversatz bezogen hat (vgl. SHukri 1950, 1951), bleibt offen mangels Vergleichsproben. Denkbar wäre jedenfalls für das jüngere Pleistozän auch eine Komponente aus den gebirgsnahen Bereichen der Küstenebene, wo alte Sande teilweise deckenartig verbreitet sind. Doch müssen auch diese Sande letztlich eine Lieferquelle gehabt haben, welche Sande mit einem instabilen Schwermineralspektrum obiger Zusammensetzung bereitzustellen vermochten. Freilich ist damit eine direkte Staubzulieferung aus den Wüsten nicht völlig ausgeschlossen, aber dagegen spricht die Schwermineral-Vergesellschaftung und die Körnungsverteilung, die wenigstens für die Hauptmasse der äolischen Sedimente auf die eiszeitliche Küstenzone weist. 
Doch ist die Kornverfeinerung landeinwärts nicht allein über eine äolische Saigerung erklärbar. Es spielt daneben der Faktor Materialbereitstellung eine Rolle. Wie die Bemerkungen zur Stratigraphie zeigen, wurde das Material aus dem jeweiligen Strandbereich während der Kaltphasen ausgeweht (vgl. auch Lietz \& Schwarzbach 1971). Darüber hinaus muß es sich aus Gründen der Pedostratigraphie bei der Materialbereitstellung um Regressionszeiten gehandelt haben, also trockene Stadiale. Die „Interstadiale“ waren hingegen Zeiten der Pedogenese.

Aber schon in der ersten Regressionsphase des frühen Würm läßt sich in Kissufim sandiger Tonlöß nachweisen. Dies bedeutet nicht grundsätzlich einen in sehr kurzer Zeit tief abgesunkenen Meeresspiegel, sondern im Prinzip nur die Trockenlegung toniger Meeressedimente. Die Grenze Sand zu Ton liegt heute vor der Küste Israels bei etwa minus $60 \mathrm{~m}$ NN (EMERy \& Bentor 1960). Doch sind dabei die Störungen zu bedenken, die durch anthropogene Eingriffe in die Umwelt des Festlandes ausgelöst werden. Deshalb kann man heute mit verstärkter Rücklieferung von Sand in den marinen Bereich rechnen, die sich bathymetrisch auswirkt. Entsprechende, aber klimatisch bedingte Mobilisierungen der Festlandsande mit fluvialer Rücklieferung zum Meer müssen auch für die relativ ariden Stadialzeiten mit absinkendem Meeresspiegel unterstellt werden (vgl. „Deluviallöß“ in BRUNNACKER 1974, 1980).

Die jeweilige Lage der marinen küstennahen Sande wurde in Regressionszeiten anfänglich rasch unterschritten und tonige Meeressedimente standen infolge Vererdung für die Auswehung als Agglomerate zur Verfügung. Im Verlauf der während der letzten Eiszeit zunehmenden Tendenz zur Absenkung des Meeresspiegels rückte die strandnahe Sandfazies ebenfalls in tiefere Niveaus hinab. Dabei hat sich diese selbstverständlich der jeweiligen Lage der regredierenden Küstenlinie angepaßt, doch mit einer gewissen Verzögerung, so daß letztlich auch bei tiefstem Meeresstrand Sand zur Auswehung kommen konnte. Neben dieser Strandzone standen selbstverständlich auch die Sande für eine Auswehung zur Verfügung, die aus dem Binnenland fluvial in die verbreiterte Küstenzone zurückgeführt wurden.

Damit muß die Kornsaigerung landeinwärts ebenfalls etwas modifiziert gesehen werden. Die Tonteilchen wurden als Agglomerate verweht. Bei der Körnungsanalyse werden sie aber aufgelöst in Einzelkörner, was zur Folge hat, daß der Anteil der Tonfraktion jetzt überproportional vertreten ist. Der sandige Tonlöß gehört bei einer solchen Betrachtung eher in den Mittel- bis Feinsandbereich, und der Tonlöß ist genetisch als schluffiger Löß zu bewerten:

Küste:

Kurkar-Sand

Binnenland:

$=$ Mittel-

Grobsand

\begin{tabular}{ll}
\multicolumn{2}{c}{ Binnenland: } \\
sandiger Tonlöß & Tonlöß \\
$=$ Fein- bis & $=$ Schluff \\
Mittelsand &
\end{tabular}

\section{Land-Meer-Beziehungen}

Nur bei Meeresregressionen stand in der jeweiligen Strandzone genügend Material für die Auswehung zur Verfügung. Dies waren die Zeiten, in denen sich größere Inland-Eismassen aufgebaut haben. Damit gibt die behandelte Äolianit-Boden-Folge die jung-pleistozänen Schwankungen des Meeresspiegels über das östliche Mittelmeer hinaus wieder.

Gegen Ende des letzten Interglazials setzte die Regression ein. Sie war in sich weiter gegliedert. So zeichnen sich frühe Schwankungen in Kissufim und Ruhama mit zwei (oder drei) Absenkungen und jeweils nachfolgendem Anstieg des Meeresspiegels ab. Dann wurde 
während einer ersten tieferen Absenkung der Kurkar I gebildet mit nachfolgender Pause, welche durch den mittelpaläolithischen Boden gekennzeichnet ist. Der Kurkar II und seine z. T. sehr feinkörnigen Äquivalente vertreten die tiefste Absenkung. Die anschließende Ingression wird durch den epipaläolithischen Boden signalisiert. Der küstennahe, nur z. T. vertretene Kurkar III weist wiederum auf eine Unterbrechung der Ingression bei relativ hohem Meeresspiegel während der jüngeren Tundrenzeit hin. Allerdings darf daraus nicht geschlossen werden, daß die Auswehung entlang der jeweiligen Küstenlinie allzu streng an die Regressionen gebunden war; denn einzelne Profile (nördlich Tel Aviv: Hefziba und Natanya) zeigen vielleicht das Fortleben der Sandauswehungen mit nur kürzeren Unterbrechungen während des unterstellten Meeresspiegelanstieges an.

\section{Stratigraphie}

Eine stratigraphische Korrelation einerseits mit der marinen Entwicklung und andererseits mit dem periglazialen Raum nördlich der Alpen liegt nahe. Jedoch stehen ihr erhebliche Schwierigkeiten entgegen, handelt es sich doch um ganz verschiedene Faziesbereiche. Deshalb ist erforderlich, soweit möglich, eine absolute Zeitskala zu benutzen. Für die jüngsten Abschnitte können Radiokarbon-Daten herangezogen werden. Für das ältere Jungpleistozän genügen dieselben jedoch nicht mehr, da die Differenz zwischen $\mathrm{C}^{14}$ Jahren und Erdjahren zu groß wird, die Fehlerbreite der Daten sich vergrößert und schließlich die Grenze des Meßbereiches erreicht wird (vgl. Grootes 1977). Hier bietet sich die Th230/U234-Methode an.

Abgesehen von einem Beach-rock mit einem Th/U-Alter um 80000 Jahre liegt eine Reihe solcher Werte aus Travertinen und Höhlensintern aus Israel vor (SCHWARCZ et al. 1979, 1968). Darüber hinaus gibt es weit über tausend Th/U-Datierungen aus dem marinen und terrestrischen Raum. Sie werden derzeit von unserer Kölner Arbeitsgruppe statistisch für eine Publikation vorbereitet (Hennig, Grün, Chai und Brunnacker). Aus technischen Gründen wurden jedoch in Abb. 11 nur zusätzliche Werte von HeNNIG (1979) aufgenommen (vgl. auch Grün, Brunnacker und Hennig 1982). Auch die Daten von Strandterrassen und Riffen (z. B. Bloom et al. 1974) werden in die angekündigte Zusammenstellung einbezogen, so daß sie ebenfalls an dieser Stelle im wesentlichen unberücksichtigt bleiben können - zumal hier ein weiteres Problem hereinspielt. Dennoch steht anhand der in Abb. 11 angeführten Werte bereits eine recht brauchbare Information für den Umkreis des letzten Interglazials und die Würmeiszeit zur Verfügung. Diese kann allerdings aus den oben genannten Gründen vorerst nur sehr eingeschränkt mit den Radiokarbon-Daten in Verbindung gebracht werden.

\subsection{Mediterraner Bereich}

Abgesehen von Profilen des Typ Kitros wurden bei eigenen Untersuchungen über den Löß im mediterranen Raum (BRUNNACKER 1974) hochglaziale Lößabschnitte und deren regionale Differenzierung genauer zu fassen versucht - was Ziel der damaligen vorzugsweise paläoklimatisch orientierten Untersuchungen war.

Das letzte Interglazial und das frühe Würm sind nun durch die Untersuchungen zwischen Gaza und Beer Sheva ebenfalls gut bekannt (Abb. 10 u. 11). Sie zeigen einen Pedokomplex, der bis vier Paläoböden umfaßt und damit die früheren Befunde insofern bestätigt, als die, aber nur scheinbar intensiv ausgeprägten Böden des Hamra-Types in das frühe Würm hineinreichen und darüber hinaus erneut im mittleren Würm wie gegen Ende dieser Eiszeit auftreten. Pedostratigraphie ist keine Chronostratigraphie (BRUNNAcker 1979). 


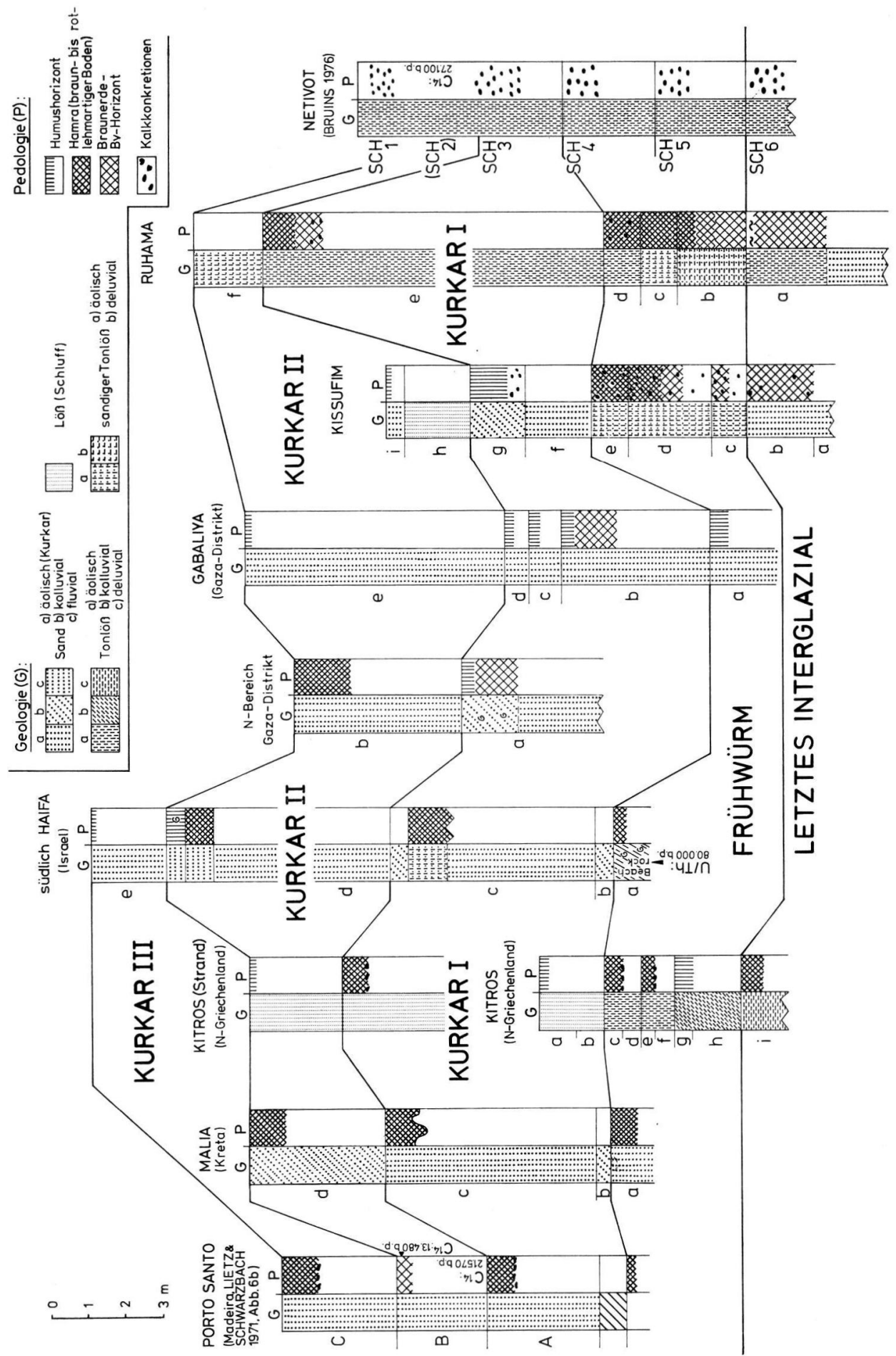

Abb. 10: Korrelation des Jungpleistozäns im mediterranen Raum. 


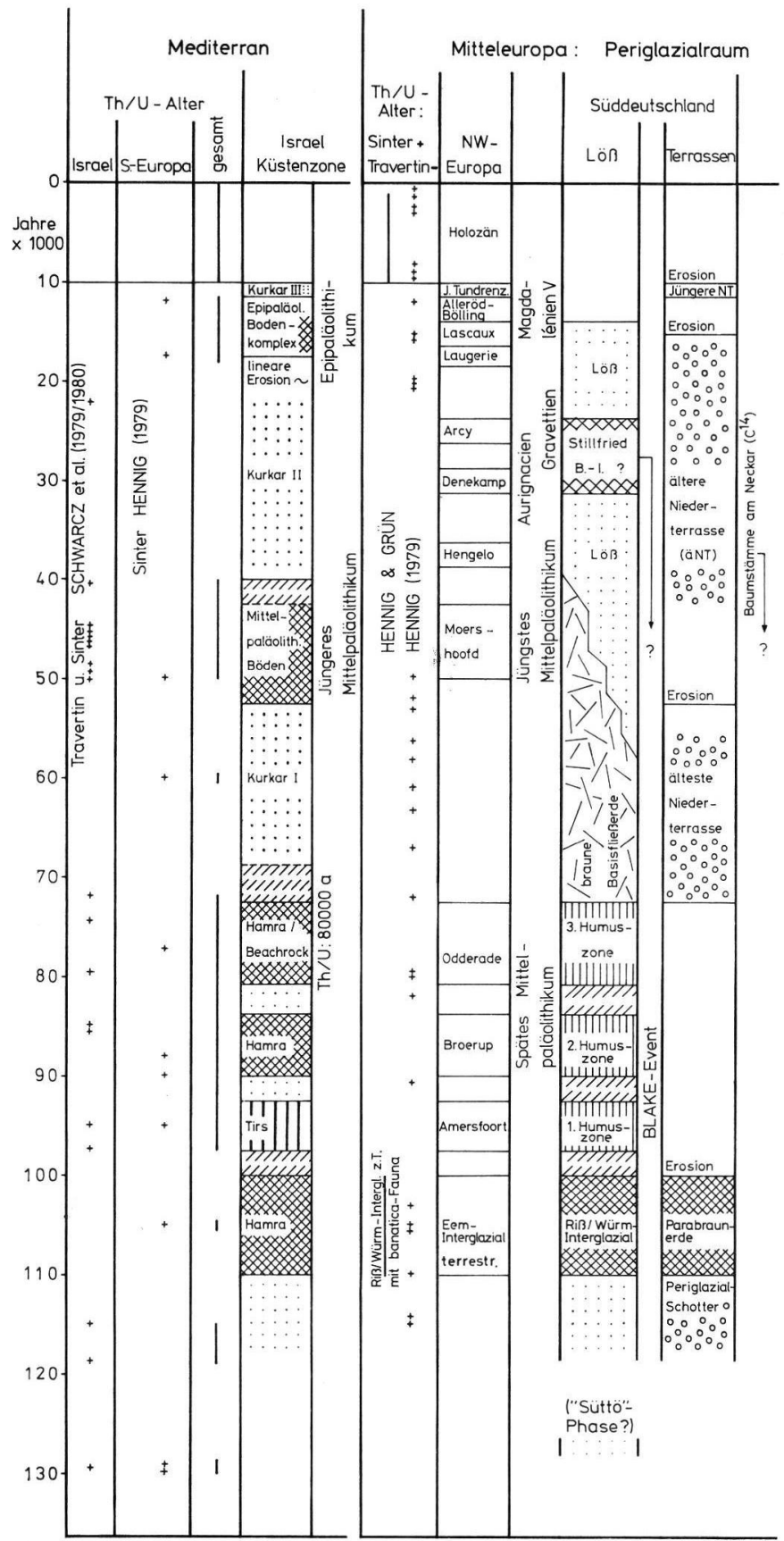

Sommertemperatur (Juli)

NW-Europa Mediterran

•C: $\begin{array}{rlllll}\sim 5 & \sim 10 & \sim 15 & \sim 20 & \sim 25 & \sim 30\end{array}$

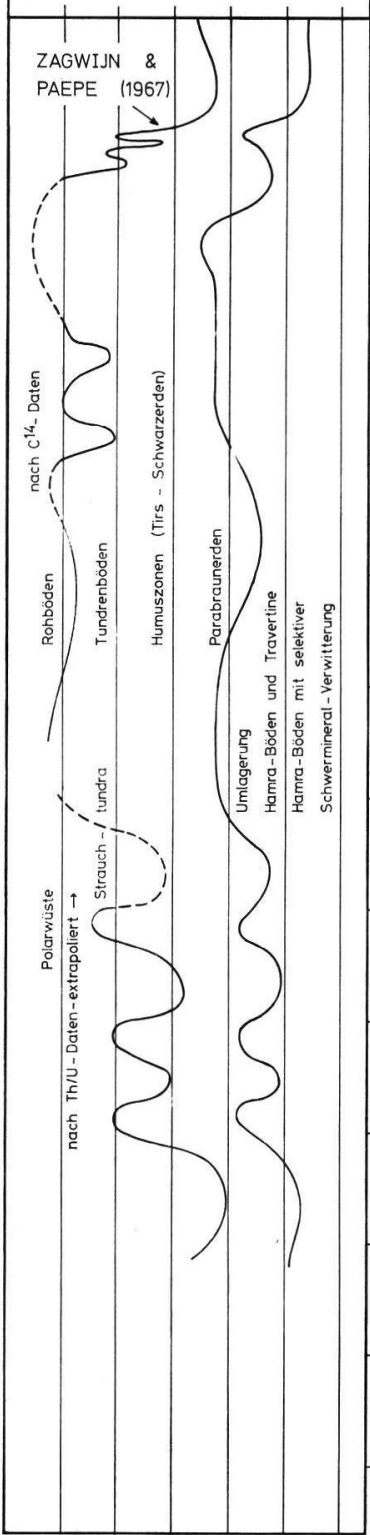

Abb. 11: Stratigraphische Korrelation des Jungpleistozäns: Mediterran - Mitteleuropa. 
Th/U-Daten aus Kalkabsätzen in Israel häufen sich um 10000 bis 80000 Jahre sowie um 50000 Jahre. Etwas feuchtere Bedingungen lassen sich daraus ableiten. Auf dieser Grundlage ergibt sich die in Abb. 11 gebrachte Einstufung.

\subsection{Mariner Bereich}

Th/U-Daten von jungpleistozänen Strandterrassen stellen die Beziehung zur TiefseeAbfolge her. Fügt man die von Shackleton \& Opdyke (1973) bzw. Emiliani \& ShackLETON (1974) herausgearbeitete Kurve über die Schwankungen des delta O18 in unser Zeitschema, dann ergeben sich insofern Probleme, als das 5e-Stadium mit 125000 Jahren älter ist als unser letztes Interglazial (bezogen auf Travertine). Doch ist dabei zu bedenken, daß zumindest im südöstlichen Mitteleuropa im Löß bzw. Schwemmlöß unmittelbar unter dem R/W-Boden bereits die hochinterglaziale banatica-Fauna bzw. die Süttö-Phase (Abb.11) auftritt (BrunNaCKer et al. 1980). Die einzige, aber nach dem derzeitigen Kenntnisstand nicht sehr wahrscheinliche Alternative besteht darin, daß aus methodischen Gründen grundsätzliche Altersunterschiede zwischen den Bildungen des marinen und des terrestrischen Raumes bestehen.

\subsection{Periglazialraum}

Im periglazialen Raum nördlich der Alpen gibt es verschiedene Faziesbereiche, die sich wohl zu einem stratigraphischen Verband zusammenfassen lassen, aber hinsichtlich der Einzelheiten noch immer erhebliche Korrelationsprobleme aufwerfen.

\subsubsection{E e m}

In Nordwesteuropa kann vom Eem (rd. 10000 Jahre Dauer - MülleR 1974) ausgegangen werden. Das marine Eem wird von MANGERUd et al. (1979) mit dem Stadium 5e der Tiefsee-Abfolge verknüpft. Hier steht die Frage im Raum, ob das marine und das terrestrische Eem wirklich stratigraphisch ident sind. Die aus dem Hangenden des Eem bekannten, pollenanalytisch erfaßten Interstadiale, nach oben ergänzt durch C14-Werte, lassen sich prinzipiell mit der Abfolge in Israel zusammenfügen, wenn von der Zahl der Klimaschwankungen und deren Größenordnung ausgegangen wird (Abb. 11).

\subsubsection{Flie $B$ erde-Lö $B-F$ olge}

Die reliefabhängige Fließerde-Löß-Paläoboden-Folge birgt auf den ersten Blick ebenfalls keine gravierenden Unterschiede gegenüber Israel, zumal der Blake-Event im untersten Bereich der Humuszonen des frühen Würm zu suchen ist (KuKLA \& Kočr 1972). Brauchbare (?) Radiokarbon-Daten stehen zumeist in Zusammenhang mit Kulturschichten des jüngeren Paläolithikums im höheren Würm (vgl. BrunNacker \& HaHN 1978).

\subsubsection{Travertine}

Wesentliche Schwierigkeiten tauchen allerdings dann auf, wenn Th/U-Daten von solchen Travertinen einbezogen werden, die konventionellerweise dem Riß/Würm-Interglazial (z. B. in Bad Cannstatt - Reiff 1965) zuzuordnen sind. Nach neueren Befunden liegen derartige Alterswerte lediglich um und etwas über 100000 Jahren. Bei den Höhlensintern ist hingegen die Streubreite größer und reicht bis etwa 50000 Jahre (HeNNIG 1979) (Abb. 11). 


\subsubsection{Terrassen}

Ebenso große Schwierigkeiten treten bei den Terrassen des Alpenvorlandes auf - ein Thema, das hier nur gestreift werden kann. Die Hochterrasse im Sinne von PENCK gehört sicherlich normalerweise in die Riß-Eiszeit. Doch gibt es bei München (M. BRUNNACKer \& K. Brunnacker 1962) und am Lech (Tillmanns et al. 1982) eine Unterteilung des Hochterrassenkörpers durch einen (aufgearbeiteten) Hochflutlehm mit z. T. interglazialer Auewald-Molluskenfauna. Darüber folgt ein Periglazialschotter mit bereichsweise erheblicher kryoturbater Uberprägung und synsedimentären Eiskeilen. Den Abschluß bildet ein Boden, der konventionellerweise dem Riß/Würm-Interglazial zugehört. Die Auffassung von Frenzel (1973) wie auch von Kukla (1977) über offene Zeitbeziehungen zwischen dem Riß/Würm-Interglazial und dem Eem-Interglazial ist damit nicht ganz abwegig.

Ein Punkt, der die Terrassenstratigraphie innerhalb des periglazialen Würm und die Befunde im mediterranen Bereich etwas annähert, ist der (lokale) Nachweis einer „ältesten“ Niederterrasse (Tillmanns et al. 1981), die jünger ist als das Frühwürm. Hinzu kommt die von Löscher et al. (1980) beschriebene Anreicherung von Baumstämmen im Neckar-Schwemmfächer um 40000-30000 Jahre vor heute, also vor und zu Beginn der Ablagerung der älteren (= Haupt-) Niederterrasse (Abb. 11). Problemlos ist hingegen die Einstufung der jüngeren Niederterrasse in die Jüngere Tundrenzeit (Thoste 1974; BRUNNACKER 1978).

Die prähistorischen Funde zeigen in Mitteleuropa einmal eine Häufung im frühen Würm, eine zweite im Bereich Moershoofd- bis Arci-Interstadial sowie schließlich eine dritte im Zeitbereich Bölling bis Jüngere Tundrenzeit (mdl. Mitt. von Kollegen Bosinski). Daraus darf gefolgert werden, daß die Zwischenzeiten für den Menschen zum Aufenthalt im periglazialen Raum Mitteleuropas nicht sonderlich lebensgünstig waren (Abb. 11).

\section{Klimaentwicklung}

Fernziel der stratigraphischen Vergleiche muß die Klärung der Klimaentwicklung des jüngeren Pleistozäns sein. Dies gilt einmal für die Meer-Land-Situation als die Möglichkeit, die globale Entwicklung zu fassen. Sie wird letztlich über die Entwicklung auf den Festländern durch das Verhalten der Inlandeismassen angezeigt. Zum anderen steht auf den Festländern selbst die Überbrückung von Klimazone zu Klimazone im Vordergrund. Dabei kann davon ausgegangen werden, daß kältere Zeiten durch Solifluktion und Löß bzw. in dem behandelten Raum von Israel durch äolische und fluviale Vorgänge gekennzeichnet sind. Die wärmeren, vor allem etwas feuchteren Abschnitte auch innerhalb des Würm waren durch Pedogenese geprägt. Auf der Grundlage der Böden ist folgende Klassifikation möglich:

\section{Küstenebene von Israel}

1. Hamra mit deutlicher Schwermineralverwitterung; Kalkausscheidung in Form von Höhlensinter und Travertin = letztes Interglazial

2. Hamra ohne deutliche Schwermineralverwitterung; jedoch Sinter- und Travertin = wärmere Abschnitte des frühen und mittleren Würm

3. Hamra ohne Schwermineralverwitterung; ohne Sinter sowie ohne Travertin (vielleicht bisher nicht bekannt) $=$ Spätglazial. 


\section{Periglazialraum nördlich der Alpen}

1. Parabraunerde und Höhlensinter sowie Travertin z. T. mit banatica-Fauna = letztes Interglazial

2. Humuszonen (Schwarzerden bis tirsoide Böden), Höhlensinter $=$ frühes Würm

3. Dünne Sinterlagen in Höhlen $=$ mittleres Würm

4. Tundrenböden (Gleye und braunerdeartige Böden) $=$ besonders im höheren Würm.

Die grundsätzlichen Daten über die Temperaturdepression im Periglazialraum lassen sich von ZAGWIJN \& PAEPE (1968) übernehmen. Noch an der Neretva in Jugoslawien ist anhand der Molluskenfauna eine entsprechende Erniedrigung nachweisbar (BRUNNACKER et al. 1969). Noch weiter südlich, im eumediterranen Bereich, lassen sich vorerst keine eigenerı genaueren Befunde anführen, wenn von der Aussage abgesehen wird, daß dort die letzteiszeitlichen Molluskenfaunen auf ein rauhes Klima weisen. Deshalb wurden die Angaben von FARRAND (1971) übernommen, wonach im Vorderen Orient die letzteiszeitliche Temperaturdepression bei $5-7^{\circ} \mathrm{C}$ gelegen hat. Dem stehen im nördlich davon gelegenen Periglazialraum $12-15^{\circ} \mathrm{C}$ Temperaturerniedrigung gegenüber.

Auf dieser Grundlage ergibt sich der in Abb. 11 vergleichend dargestellte Klimaverlauf beider Räume, wobei allerdings im Periglazialraum die C14-Daten im Bezug auf die $\mathrm{Th} / \mathrm{U}-\mathrm{Daten}$ etwas zu jung erscheinen.

Ein weiteres Problem deutet sich für die Temperaturen im östlichen Mittelmeer an. Für das Hochglazial werden für dessen Oberflächenwasser rd. $18^{\circ} \mathrm{C}$ (im Winter) und $25^{\circ} \mathrm{C}$ (im Sommer) veranschlagt im Unterschied zum westlichen Mittelmeer mit $7^{\circ} \mathrm{C}$ bzw. $15^{\circ} \mathrm{C}$ (THIEDE1980). Ahnlich geringe Temperaturabsenkungen werden für den Golf von Akaba genannt (Reiss et al. 1980).

Zusammenfassend läßt sich sagen, daß viele gemeinsame Züge zwischen der eiszeitlichmediterranen und der periglazialen Entwicklung vorhanden sind. Bezüglich der Einzelheiten taucht aber eine Reihe von Problemen auf, für die allein Lösungsansätze vorhanden sind. Dabei muß auch die Stellung des Stillfried B erneut überdacht werden.

Die höchst unterschiedliche Bodenausprägung nördlich und südlich der Alpen während der Würm-Eiszeit läßt sich durch folgende Gegebenheiten erklären:

1. Relativ gesehen war der eiszeitlich mediterrane Raum immer wärmer als der periglaziale Raum.

2. Die eiszeitliche Temperaturdepression im mediterranen Bereich war geringer als im Periglazialraum. Auch bei nur geringer Erwärmung wurden die Schwellenwerte im mediterranen Raum rascher überschritten, z. B. Bodenausbildung und Sinter, als im Periglazialraum.

3. Aus der Sicht des Klimatyps war der eiszeitlich-mediterrane Raum der gleiche wie heute (BRUNNACKER 1979).

4. Der Klimagang im Ablauf der Würm-Eiszeit zeigt sowohl nördlich wie südlich der Alpen gleichartige Züge. Allerdings wirkt sich im höheren Würm die eiszeitliche Temperaturdepression nördlich der Alpen stärker aus als südlich der Alpen.

5. Generell waren die Stadialzeiten trockenere Abschnitte. Die Interglaziale und Interstadiale sind hingegen als relativ feucht ausgewiesen. Der periglaziale Löß steht nördlich der Alpen im direkten Zusammenhang mit den trockenen Stadialen, besonders im höheren Würm. Im mediterranen Bereich besteht hingegen eine indirekte Beziehung zwischen den Äolianiten der Küstenzone und der Klimaentwicklung. Hier ist die eiszeitliche Küstenlinie Hauptlieferant für äolischen Sand bis Schluff. Die Auswehung erfolgte in den Regressionszeiten, also während des Aufbaues von Inlandeismassen und damit letztlich synchron der Phase der Lößbildung im Periglazialraum. 


\section{Schriftenverzeichnis}

Bloom, A. L., Broeker, W. S., Chappell, J. M. A., Matthews, R. K. \& Mesolella, K. J. (1974): Quarternary Sea Level Fluctuations on a Tectonic Coast: New $230 \mathrm{Th} / 234 \mathrm{U}$ Dates from the Huon Peninsula, New Guinea. - Quarternary Research, 4: 185-205, 5 Abb., 4 Tab.; Washington.

Bruins, H. J. (1976): The Origin, Nature and Stratigraphy of Paleosols in the loessial Deposits of the NW-Negev (Netivot, Israel). - Thesis Hebrew Univ. Jerusalem: 155 S.; Jerusalem.

BrunNaCker, K. (1958): Zur Parallelisierung des Jungpleistozäns in den Periglazialgebieten Bayerns und seiner östlichen Nachbarländer. - Geol. Jb., 76: 129-150, 5 Abb., 3 Tab.; Hannover.

- (1974): Lösse und Paläoböden der letzten Kaltzeit im mediterranen Raum. - Eiszeitalter und Gegenwart, 25: 62-93, 10 Abb., 3 Tab.; Ohringen.

- (19/8): Der Niederrhein im Holozän. - Fortschr. Geol. Rheinld. u. Westf., 28: 399-440, 14 Abb., 4 Tab., 1 Taf.; Krefeld.

- (1979): Zur Bodengeschichte des Jungquartärs im mediterranen Raum. - Z. Geomorph. N. F., Suppl. Bd. 33: 109-117, 3 Abb.; Berlin - Stuttgart.

- (1980): Young Pleistocene Loess as an Indicator for the Climate in the Mediterrannean Area. - Palaeoecology of Africa and the Sourrounding Islands, 12: 99-113, 6 Abb., 1 Tab.; Rotterdam.

- , Altemüller, H.-J. \& Beug, H.-J. (1969): Das Profil von Kitros in Nord-Griechenland als Typusprofil einer mediterranen Lößprovinz. - Eizeitalter u. Gegenwart, 20: 90-110, 14 Abb., 3 Tab.; O'hringen.

- , Basler, D., Ložek, V., Beug, H.-J. \& Altemüller, H.-J. (1969): Zur Kenntnis der Lösse im Neretva-Tal. - N. Jb. Geol. Paläont. Abh., 132: 127-154, 3 Abb., 2 Tab., Taf. 10-14; Stuttgart.

- \& HAHN, J. (1978): Der jungpleistozäne Löß samt paläolithischen Kulturen in den Rheinlanden als Glied einer zeitlichen und räumlichen Faziesänderung. - Beiträge zur Quartärund Landschaftsforschung - Festschr. z. 60. Geburtstag von Julius Fink: 37-51, 2 Abb.; Wien (Hirt).

- , Janossy, D., Krolopp, E., Skoflek, I. \& Urban, B. (1980): Das jungmittelpleistozäne Profil von Süttö 6 (Westungarn). - Eiszeitalter u. Gegenwart, 30: 1-18, 5 Abb., 2 Tab., Hannover.

Brunnacker, M. \& BrunnaCker, K. (1962): Weitere Funde pleistozäner Molluskenfauna bei München. - Eiszeitalter u. Gegenwart, 13: 129-137, 4 Tab.; OOhringen.

DAN, J. \& YAALON, D. H. (1971): On the origin and nature of the paleopedological formations in the coastal desert fringe areas of Israel. - Paleopedology: 245-260; Jerusalem.

Emerr, K. O. \& Bentor, Y. K. (1960): The Continental Shelf of Israel. - Geol. Survey, 26: 25-42, 5 Abb.; Jerusalem.

- \& Neev, D. (1960): Mediterranean Beaches of Israel. - Geol. Survey, 26: 1-24, 4 Abb., 1 Tab., 1 Anhang; Jerusalem.

Emiliani, C. \& Shackleton, N. J. (1974): The Brunhes Epoch: Isotopic Paleotemperatures and Geochronology. - Science, 183: 511.

FARRAND, W. R. (1971): Late Quaternary Paleoclimates of the Eastern Mediterranean Area. In: Turekian, K. K.: The late Cenozoic Glacial Ages: 529-564, 12 Abb., 2 Tab.; New Haven.

Frenzel, B. (1973): Some Remarks on the Pleistocene Vegetation. - Eiszeitalter u. Gegenwart, 23/24: 281-292, 1 Abb.; OOhringen.

Grootes, P. M. (1977): Thermal Diffusion Isotopic Enrichment and Radiocarbon Dating beyond 50.000 Years B. P. - Rijks-Univers. Groningen, Proefschrift: 221 S.; Groningen.

Grün, R., Brunnacker, K. \& Hennig, G. J. (1982): 230Th/234U-Daten mittel- und jungpleistozäner Travertine im Raum Stuttgart. - Jber. Mitt. oberrh. geol. Ver., N. F., 64: 201-211, 3 Abb., 1 Tab.; Stuttgart.

HenNig, G. J. (1979): Beiträge zur Th-230/U-234-Altersbestimmung von Höhlensintern sowie ein Vergleich der erzielten Ergebnisse mit denen anderer Absolutdatierungsmethoden. - Diss. Univ. Köln: 173 S. + XXVII S., 27 Diagramme, Tab. A-G; Köln.

KuKLA, G. J. (1977): Pleistocene land-sea correlations. - Earth-Sci. Rev., 13: 307-374, 21 Abb.; Amsterdam.

KuKLA, G. J. \& Koči, A. (1972): End of the last Interglacial in the Loess Record. - Quaternary Research, 2: 374-383, 7 Abb.; Washington. 
Lietz, J. \& Schwarzbach, M. (1971): Quartäre Sedimente auf der Atlantik-Insel Porto Santo (Madera-Archipel) und ihre paläoklimatische Deutung. - Eiszeitalter u. Gegenwart, 22: 89-109, 10 Abb.; Óhringen.

Löscher, M., Becker, B., Bruns, M. Hieronymus, U., Mäusbacher, R., Münnich, M., Münzing, K. \& Schedler, J. (1980): Neue Ergebnisse über das Jungquartär im Neckar-Schwemmfächer bei Heidelberg. - Eiszeitalter u. Gegenwart, 30: 89-100, 3 Abb., 4 Tab., 1 Taf.; Hannover.

Mangerud, J., Sønstegaard, E. \& Sejrup, H.-P. (1979): Correlation of the Eemian (interglacial) Stage and the deepsea oxygen-isotope stratigraphy. - Nature, 277: 189-192, 3 Abb.; London.

MüLLER, H. (1974): Pollenanalytische Untersuchungen und Jahresschichtenzählungen an der eemzeitlichen Kieselgur von Bispingen/Luhe. - Geol. Jb., A 21: 149-169, 5 Abb., 3 Tab.; Hannover.

Nachmais, J. (1969): Source rocks of the Saqiye Group sediments in the Coastal Plain of Israel - a heavy mineral study. - Israel J. of Earth-Sciences, 18:1-16, 5 Abb., 6 Tab.; Jerusalem.

Range, P. (1925): Das Diluvium Palästinas. — Die Eiszeit, 2: 116-118; Leipzig.

REIFF, W. (1965): Das Alter der Sauerwasserkalke von Stuttgart - Münster - Bad Cannstatt - Untertürkheim. - Jber. u. Mitt. oberrh. geol. Ver., N. F. 47: 111-134, 3 Abb., 3 Beil.; Stuttgart.

Reiss, Z., Almogi-Labin, A., Halicz, E., Winter, A., Wolf, M. \& Ross, D. A. (1980): Late Quaternary Paleoceanography of the Gulf of Aquaba (Elat), Red Sea. - Quaternary Research, 14: 294-308, 7 Abb., 1 Tab.; Washington.

Ronen, A., Gilead, D., Shachnai, E. \& Saul, A. (1972): Upper Acheulean in the Kissufim Region. - Proc. American Philosoph. Soc., 116: 68-92, 33 Abb.; Philadelphia.

Schwarcz, H. P., Blackwell, B., Goldberg, P. \& Marks, A. E. (1979): Uranium series dating of Travertine from archaeological sites, Nahal Zin, Israel. - Nature, 277: 558-560, 2 Abb., 1 Tab.; London.

- , Goldberg, P. D. \& Blackwell, B. (1980): Uranium series Dating of Archaeological Sites in Israel. - Israel J. of Earth-Sciences, 29: 157-165, 5 Abb., 1 Tab.; Jerusalem.

Shackleton, N. J. \& Opdyke, N. D. (1973): Oxygen Isotope and Palaeomagnetic Stratigraphy of Equatorial Pacific Core V 28-238: Oxygen Isotope Temperatures and Ice Volumes on a 10 ' Year and 106 Year Scale. - Quaternary Research, 3: 39-55, 9 Abb., 4 Tab.; Washitgton.

SHUKRI, M. N. (1950): The mineralogy of some Nile sediments. - Quart. Journ. Geol. Soc. London, 105: 511-534; London.

- (1951): Mineral analysia tables of some Nile sediments. - Bull. Inst. Fouad 1. - Desert, 1: 39-67; Cairo.

Slatkine, A. \& Pomerancblum, M. (1958): Contributions to the study of the Pleistocene in the coastal plain of Israel: Unstable heavy minerals as criteria of depositional environment. Geol. Survey of Israel, 19: 11 S., Jerusalem.

Stearns, Ch. E. \& Thurber, D. L. (1965): Th230 - U234 dates of the late Pleistocene marine fossils from the Mediterranean and Moroccan littorals. - Quaternaria, 7: 29-42, 2 Tab.; Roma.

Thiede, J. (1980): The late Quaternary marine Paleoenvironments between Europe and Africa. - Palaeoecology of Africa, 12: 213-225, 5 Abb.; Rotterdam.

Tillmanns, W., Löscher, M., Münzing, K. \& Brunnacker, K. (1982): Die Rainer Hochterrasse zwischen Lech und Donau. - Jber. Mitt. oberrhein. geol. Ver., N. F., 64: 79-99, 9 Abb., 1 Tab.; Stuttgart.

Thoste, V. (1974): Die Niederterrassen des Rheins vom Neuwieder Becken bis in die Niederrheinische Bucht. - Diss. Univ. Köln: 130 S., 31 Abb., 6 Tab.; Köln.

Vroman, J. (1944): The petrology of sandy sediments of Palestine. - Bull. Geol. Dept. Hebrew Univ., 5: 1-11, 1 Fig., 1 Tab.; Jerusalem.

Wintle, A. G. \& Brunnacker, K. (1982): Ages of volcanic tuff in Rheinhessen obtained by thermoluminiscence dating of the loess in which they occur. - Die Naturwissenschaften, 69: 181-182, 1 Abb., 1 Tab.; Heidelberg.

YAALON, D. H. (1969): Origin of desert loess (abs.). - In: Etudes sur le Quaternaire dans le Monde; 8th INQUA Cong., 2: 755; Paris.

- \& DAN, J. (1974): Accumulation and distribution of loess-derived deposits in the semi-desert and desert fringe areas of Israel. - Z. Geomorph. N. F., Suppl. Bd. 20: 91-105, 2 Abb., 2 Phot.; Berlin.

ZAGwiJn, W. \& PAEPE, R. (1968): Die Stratigraphie der weichselzeitlichen Ablagerungen der Niederlande und Belgiens. - Eiszeitalter u. Gegenwart, 19: 129-146, 6 Abb.; Ohringen. 